Pacific

Journal of

Mathematics

AN ORLIK-RAYMOND TYPE CLASSIFICATION OF SIMPLY CONNECTED 6-DIMENSIONAL TORUS MANIFOLDS WITH VANISHING ODD-DEGREE COHOMOLOGY

SHINTARÔ KUROKI 


\title{
AN ORLIK-RAYMOND TYPE CLASSIFICATION OF SIMPLY CONNECTED 6-DIMENSIONAL TORUS MANIFOLDS WITH VANISHING ODD-DEGREE COHOMOLOGY
}

\author{
SHINTARÔ KUROKI
}

Dedicated to Professor Mikiya Masuda on his 60th birthday.

\begin{abstract}
The aim of this paper is to classify simply connected 6-dimensional torus manifolds with vanishing odd-degree cohomology. It is shown that there is a one-to-one correspondence between equivariant diffeomorphism types of these manifolds and 3-valent labelled graphs, called torus graphs, introduced by Maeda, Masuda and Panov. Using this correspondence and combinatorial arguments, we prove that a simply connected 6-dimensional torus manifold with $H^{\text {odd }}(M)=0$ is equivariantly diffeomorphic to the 6-dimensional sphere $S^{6}$ or an equivariant connected sum of copies of 6dimensional quasitoric manifolds or $S^{4}$-bundles over $S^{2}$.
\end{abstract}

\section{Introduction}

Let $M$ be a $2 n$-dimensional closed, connected, oriented manifold with an effective $n$-dimensional (i.e., half-dimensional) torus $T^{n}$-action. We call $M$, or $(M, T)$, a torus manifold if $M^{T} \neq \varnothing$ (see [Hattori and Masuda 2003]), where $M^{T}$ is the set of fixed points. A toric manifold (i.e., a nonsingular, complete toric variety viewed as a complex analytic space) with restricted $T^{n}$-action is a typical example of a torus manifold. Recall that a toric manifold is a complex $\left(\mathbb{C}^{*}\right)^{n}$-manifold with a dense orbit (see [Oda 1988; Fulton 1993]), and $T^{n}$ is the maximal compact subgroup of $\left(\mathbb{C}^{*}\right)^{n}$. A fundamental result of toric geometry tells us that there is a oneto-one correspondence between toric manifolds and combinatorial objects called fans. Thus, topological (more precisely, geometric) invariants of toric manifolds can be described in terms of combinatorial invariants of fans, such as equivariant cohomology rings, equivariant characteristic classes and other topological invariants.

The author was partially supported by JSPS KAKENHI grant number 15K17531, 24224002 and the JSPS Strategic Young Researcher Overseas Visits Program for Accelerating Brain Circulation "Deepening and Evolution of Mathematics and Physics, Building of International Network Hub based on OCAMI".

MSC2010: primary 57S25; secondary 94C15.

Keywords: torus manifold, torus graph, GKM graph, equivariant connected sum. 
Hattori and Masuda [2003] introduced a torus manifold as the topological generalization of a toric manifold. They also introduced the combinatorial objects called multifans (see [Masuda 1999; Hattori and Masuda 2003]), and computed topological invariants (such as equivariant characteristic classes or Todd genus for unitary torus manifolds) in terms of multifans. However, unlike the case for toric geometry, a multifan does not contain enough information to determine some topological invariants of torus manifolds (e.g., equivariant cohomology). So, in 2007, Maeda, Masuda and Panov introduced combinatorial objects called torus graphs, which were motivated by the GKM graphs introduced by Guillemin and Zara [2001]. The combinatorial information of torus graphs can completely determine the equivariant cohomology rings of torus manifolds with vanishing odd-degree cohomology, i.e., $H^{\text {odd }}(M ; \mathbb{Z})=0$ (in this paper, we only consider integer coefficients); see [Masuda and Panov 2006; Maeda et al. 2007], and see also Section 3 in this paper about torus graphs. However, in general, there is no one-to-one correspondence between torus manifolds with $H^{\text {odd }}(M)=0$ and torus graphs.

So, we are naturally led to ask the following two questions: (1) Which subclasses of torus manifolds are completely determined by combinatorial objects (like multifans or torus graphs)? (2) If we find such a subclass of torus manifolds, how can we classify such torus manifolds? Several mathematicians have answered the first question: for example, Davis and Januszkiewicz [1991] for the subclass called quasitoric manifolds (see [Buchstaber and Panov 2002] or Section 4C in this paper), Ishida, Fukukawa and Masuda [2013] for the subclass called topological toric manifolds, and Wiemeler [2013] for the class of simply connected 6-dimensional torus manifolds with $H^{\text {odd }}(M)=0$ (see Theorem 2.7). The aim of this paper is to answer the second question for the class of simply connected 6-dimensional torus manifolds with $H^{\text {odd }}(M)=0$ using torus graphs.

Let us briefly recall the classification results for torus manifolds with lower dimensions. If $T^{1}$ acts on a compact 2-dimensional manifold $M$, then $M$ is the 2-dimensional sphere $S^{2}$, the 2-dimensional real projective space $\mathbb{R} P^{2}$, the 2dimensional torus $T^{2}$ or the Klein bottle. Because $M^{T} \neq \varnothing$ and $M$ is oriented, $M$ must be equivariantly diffeomorphic to $S^{2}$ with $T^{1}$-action (see [Kawakubo 1991]). When $\operatorname{dim} M=4$, by Orlik and Raymond's theorem [1970], we have the following:

Theorem 1.1 (Orlik-Raymond). Let $M$ be a 4-dimensional simply connected torus manifold. Then, $M$ is equivariantly diffeomorphic to the 4-sphere $S^{4}$ or an equivariant connected sum of copies of complex projective spaces $\mathbb{C} P^{2}, \overline{\mathbb{C} P^{2}}$ (reversed orientation) or a Hirzebruch surface $H_{k}$.

Here a Hirzebruch surface $H_{k}(k \in \mathbb{Z})$ is a manifold which is defined by the projectivization of the complex 2-dimensional vector bundle $\gamma^{\otimes k} \oplus \epsilon$ over $\mathbb{C} P^{1}$, where $\gamma$ and $\epsilon$ are the tautological and the trivial complex line bundles over $\mathbb{C} P^{1}$. 
In this paper, we prove an Orlik-Raymond type theorem similar to Theorem 1.1 for simply connected 6-dimensional torus manifolds with $H^{\text {odd }}(M)=0$. Before we state our main results, we introduce the result for torus manifolds that are not simply connected. One of the consequences of Masuda and Panov's theorem (see Theorem 2.2 in Section 2B) is the following proposition (see also [Wiemeler 2013]).

Proposition 1.2. Let $W$ be a 6-dimensional torus manifold with $H^{\text {odd }}(W)=0$ (it might not be simply connected). Then, there are a simply connected 6-dimensional torus manifold $M$ with $H^{\text {odd }}(M)=0$ and a homology 3-sphere $h S^{3}$ such that

$$
W \cong M \#_{T}\left(h S^{3} \times T^{3}\right)
$$

up to equivariant diffeomorphism.

Here the product manifold $h S^{3} \times T^{3}$ is the product of $h S^{3}$ and the 3-dimensional torus $T^{3}$ with the free $T^{3}$-action on the second factor, and the symbol $\#_{T}$ represents the equivariant gluing along two free orbits of $M$ and $h S^{3} \times T^{3}$.

In Proposition 1.2, because the fundamental groups $\pi_{1}(W)$ and $\pi_{1}\left(h S^{3}\right)$ are isomorphic, $W$ is simply connected if and only if $h S^{3}$ is simply connected, i.e., the standard sphere. Our main theorem is a classification of the simply connected torus manifolds that appear in Proposition 1.2.

Theorem 1.3. Let $M$ be a simply connected 6-dimensional torus manifold with $H^{\text {odd }}(M)=0$. Then, $M$ is equivariantly diffeomorphic to the 6-sphere $S^{6}$ or obtained by an equivariant connected sum of copies of 6-dimensional quasitoric manifolds or $S^{4}$-bundles over $S^{2}$ equipped with the structure of a torus manifold.

This type of classification, i.e., classification by equivariant connected sum, may be regarded as the 6-dimensional analogue of Orlik and Raymond's classification in Theorem 1.1. So, in this paper, we call this theorem an Orlik-Raymond type classification (see [McGavran 1976; Kuroki 2008]).

Remark 1.4. Izmestiev [2001] proved an Orlik-Raymond type classification for a class of 3-dimensional small covers (i.e., the real analogue of quasitoric manifolds; see Section 4B), called a linear model (see also [Lü and Yu 2011; Nishimura 2012]).

The organization of this paper is as follows. In Section 2, we recall the basic facts about torus manifolds. In Section 3, we do the same for torus graphs. In particular, Corollary 3.5 is the key fact used to prove Theorem 1.3. In Section 4, we introduce the torus graphs of $S^{6}$, quasitoric manifolds and $S^{4}$-bundles over $S^{2}$. These torus graphs will be the basic graphs used to classify simply connected 6-dimensional torus manifolds with $H^{\text {odd }}(M)=0$. In Section 5, we introduce the "oriented" torus graphs and translate the equivariant connected sum around fixed points of torus manifolds to the connected sum around vertices of oriented torus graphs. In Sections 6 and 7, we prove Theorem 1.3. A brief outline of the proof is as follows. By 
Corollary 3.5, there is a one-to-one correspondence between 6-dimensional simply connected torus manifolds with $H^{\text {odd }}(M)=0$ and 3-valent torus graphs. Therefore, to prove Theorem 1.3, it is enough to prove that an (oriented) torus graph can be decomposed into basic torus graphs in Section 4 by the connected sum. We prove this using combinatorial arguments.

\section{Orbit spaces of torus manifolds}

In this section, we recall some basic facts about torus manifolds (see [Masuda 1999] or [Hattori and Masuda 2003] for details).

2A. Torus manifolds. A $2 n$-dimensional torus manifold $M$ is said to be locally standard if every point in $M$ has a $T$-invariant open neighborhood $U$ which is weakly equivariantly homeomorphic to an open subset $\Omega_{U} \subset \mathbb{C}^{n}$ invariant under the standard $T^{n}$-action on $\mathbb{C}^{n}$, where two group actions $(U, T)$ and $\left(\Omega_{U}, T\right)$ are said to be weakly equivariantly homeomorphic if there is an equivariant homeomorphism from $U$ to $\Omega_{U}$ up to an automorphism on $T^{n}$ (see, e.g., [Kuroki 2011, Section 2.1] for details).

Let $M_{i}, i=1, \ldots, m$, be a codimension- 2 torus submanifold in a $2 n$-dimensional torus manifold $M$ which is fixed by some circle subgroup $T_{i}$ in $T$. Such an $M_{i}$ is a $(2 n-2)$-dimensional torus manifold with $T / T_{i}$-action, called a characteristic submanifold. Because a torus manifold $M$ is compact, the cardinality of all characteristic submanifolds in $M$ is finite. If $M$ is locally standard, each characteristic submanifold is also locally standard.

An omniorientation $O$ of $M$ is a choice of orientation for the torus manifold $M$ as well as for each characteristic submanifold. If there are just $m$ characteristic submanifolds in $M$, there are exactly $2^{m+1}$ omniorientations (see [Buchstaber and Panov 2002; Hattori and Masuda 2003]). If $M$ has a $T$-invariant almost complex structure $J$ (in this case, $M$ is automatically locally standard), then there exists the canonical omniorientation $O_{J}$ determined by $J$. We call the torus manifold $M$ with a fixed omniorientation $\mathrm{O}$ an omnioriented torus manifold and denote it by $(M, 0)$.

2B. Orbit spaces of locally standard torus manifolds. The orbit space $M / T$ of a locally standard torus manifold $M$ naturally admits the structure of a "topological" manifold with corners. We next recall the basic facts about a topological manifold with corners (cf. the definition of a smooth manifold with corners in [Lee 2013]) and introduce the structure on $M / T$.

We will use the notation

$$
[n]=\{0,1, \ldots, n\}
$$

and

$$
\mathbb{R}_{+}^{n}=\left\{\left(x_{1}, \ldots, x_{n}\right) \in \mathbb{R}^{n} \mid x_{i} \geq 0, i=1, \ldots, n\right\}
$$


Let $Q^{n}$ be an $n$-dimensional topological manifold with boundary. A chart with corners for $Q^{n}$ is a pair $\left(V, \psi_{V}\right)$, where $V$ is an open subset of $Q^{n}$ and

$$
\psi_{V}: V \rightarrow \mathbb{R}_{+}^{n}
$$

is homeomorphic from $V$ to a (relatively) open subset $\Omega_{V} \subset \mathbb{R}_{+}^{n}$. Two charts with corners $\left(V, \psi_{V}\right)$ and $\left(W, \psi_{W}\right)$ are said to be (topologically) compatible if the composition of functions $\psi_{V} \circ \psi_{W}^{-1}: \psi_{W}(V \cap W) \rightarrow \psi_{V}(V \cap W)$ is a stratapreserving homeomorphism. This implies that if $\psi_{W}(p) \in \mathbb{R}_{+}^{n}$ contains exactly $k$ zero-coordinates then $\psi_{V}(p) \in \mathbb{R}_{+}^{n}$ also contains exactly $k$ zero-coordinates for $0 \leq k \leq n$. We call the collection of compatible charts with corners $\left\{\left(V, \psi_{V}\right)\right\}$ whose domains cover $Q^{n}$ an atlas. Then, its maximal atlas is called a structure with corners of $Q^{n}$. A topological manifold with boundary together with a structure with corners is called a (topological) manifold with corners. Let $p \in Q^{n}$ be a point of an $n$-dimensional manifold with corners $Q^{n}$. For a chart $\left(V, \psi_{V}\right)$ with corners such that $p \in V$, we define $d(p) \in[n]$ to be the number of zero-coordinates of $\psi_{V}(p) \in \mathbb{R}_{+}^{n}$. By the compatibility of charts, this number is independent of the choice of a chart with corners which contains $p$. Therefore, the map $d: Q^{n} \rightarrow[n]$ is well defined. The number $d(p)$ is called the depth of $p$. We call the closure of a connected component of $d^{-1}(k), 0 \leq k \leq n$, a codimension- $k$ face. In particular, the codimension- 0 face is $Q^{n}$ itself. Moreover, codimension- 1 , codimension- $(n-1)$ and codimension- $n$ faces are called facets, edges and vertices, respectively. The set of all edges and vertices is called a one-skeleton of $Q^{n}$ (or a graph of $Q^{n}$ ). By restricting the structure with corners on $Q^{n}$ to faces, we may regard each codimension- $k$ face as an $(n-k)$-dimensional (sub)manifold with corners.

Definition 2.1 (manifold with faces). An $n$-dimensional manifold with corners $Q$ is said to be a manifold with faces (or a nice manifold with corners) if $Q$ satisfies the following conditions:

(1) For every $k \in[n]$, there exists a codimension- $k$ face.

(2) For each codimension- $k$ face $H$, there are exactly $k$ facets $F_{1}, \ldots, F_{k}$ such that $H$ is a connected component of $\bigcap_{i=1}^{k} F_{i}$; moreover, $H \cap F \neq H$ for any facet $F \neq F_{i}(i=1, \ldots, k)$.

Let $(M, T)$ be a torus manifold. When $(M, T)$ is locally standard, by the differentiable slice theorem, the orbit space $M / T$ has the structure of an $n$-dimensional manifold with faces. On the other hand, when $M$ satisfies $H^{\text {odd }}(M)=0$, its orbit space $M / T$ satisfies a stronger condition by the following theorem (see [Masuda and Panov 2006, Lemma 2.1 and Theorem 2]).

Theorem 2.2 (Masuda-Panov). Let $M$ be a $2 n$-dimensional torus manifold. Then, the following conditions are equivalent: 
(1) $H^{\text {odd }}(M)=0$.

(2) The $T$-action on $M$ is locally standard and its orbit space $M / T$ has the structure of an n-dimensional face acyclic manifold with corners.

Here, an $n$-dimensional face acyclic manifold with corners $Q$ is an $n$-dimensional manifold with faces such that all faces $F$ of $Q$ (including $Q$ ) are acyclic, i.e., $H_{*}(F) \simeq H_{0}(F) \simeq \mathbb{Z}$. For example, if $Q$ is a simply connected 3-dimensional face acyclic manifold with corners, then it is easy to check that the boundary of $Q$ is homeomorphic to the 2-sphere $S^{2}$. Moreover, in this case, we can also check that $Q$ itself is homeomorphic to the 3-dimensional disk $D^{3}$. Therefore, as one of the consequences of Theorem 2.2, we have the following corollary.

Corollary 2.3. Let $M$ be a simply connected 6-dimensional torus manifold with $H^{\text {odd }}(M)=0$. Then, its orbit space $M / T$ is homeomorphic to the 3-dimensional disk.

By the definition of a manifold with faces $Q$, we can define a simplicial poset (partially ordered set) $\mathscr{P}(Q)$, called a face poset of $Q$ (see [Masuda 2005]), to be the set of faces in $Q$ with the empty set $\varnothing$ ordered by inclusion, where $\varnothing$ is the smallest element under this ordering, say $\preceq$. We often denote the face poset structure of $Q$ by $(\mathscr{P}(Q), \preceq)$. Let $Q_{1}$ and $Q_{2}$ be $n$-dimensional manifolds with faces. We say $Q_{1}$ and $Q_{2}$ are combinatorially equivalent if their face posets $\left(\mathscr{P}\left(Q_{1}\right), \preceq_{1}\right)$ and $\left(\mathscr{P}\left(Q_{2}\right), \preceq_{2}\right)$ are isomorphic as posets (i.e., there is an order-preserving bijection between them). We denote the equivalence by $Q_{1} \approx_{c} Q_{2}$. By the definition of weakly equivariant homeomorphism, if two locally standard torus manifolds $M_{1}$ and $M_{2}$ are weakly equivariantly homeomorphic then $M_{1} / T \approx_{c} M_{2} / T$.

2C. Characteristic functions. Let $M$ be a $2 n$-dimensional locally standard torus manifold. By the argument demonstrated in Section 2B, the orbit map $\pi: M \rightarrow$ $M / T=Q$ may be regarded as the projection onto some manifold with faces $Q$. Let $\mathscr{F}(Q)=\left\{F_{1}, \ldots, F_{m}\right\} \subset \mathscr{P}(Q)$ be the set of all facets in $Q$. By the definition of facet $F_{i} \in \mathscr{F}(Q)$, its preimage $\pi^{-1}\left(F_{i}\right)$ is a characteristic submanifold $M_{i}$. Then, there exists the circle subgroup $T_{i}(\subset T)$ fixing $M_{i}=\pi^{-1}\left(F_{i}\right)$ (recall that $\operatorname{dim} M_{i}=2 n-2$ ). Recall that $T_{i}$ is determined by a primitive element in $\mathfrak{t}_{\mathbb{Z}} \simeq \mathbb{Z}^{n}$ (the lattice of the Lie algebra of $T$ ). Therefore, using this primitive element (up to sign) in $\mathfrak{t}_{\mathbb{Z}}$, we can define the map

$$
\lambda: \mathscr{F}(Q) \rightarrow \mathfrak{t}_{\mathbb{Z}} /\{ \pm 1\},
$$

where $\mathfrak{t}_{\mathbb{Z}} /\{ \pm 1\}$ represents the quotient of $\mathfrak{t}_{\mathbb{Z}}$ by signs. We call $\lambda$ a characteristic function.

Now the choice of omniorientation 0 of $M$ determines the sign of $\lambda$ as follows. Fix an omniorientation 0 of $M$. Namely, we fix the orientation of the tangent bundle 
of $M$ (resp. $M_{i}$ ), say $\tau$ (resp. $\tau_{i}$ ). Restricting $\tau$ to the submanifold $M_{i}$, say $\left.\tau\right|_{M_{i}}$, we obtain the $T^{n}$-equivariant decomposition $\left.\tau\right|_{M_{i}} \simeq \tau_{i} \oplus v_{i}$, where $v_{i}$ is the $T_{i}$ equivariant normal bundle of $M_{i}$. Therefore, because we fix the orientation of $\left.\tau\right|_{M_{i}}$ (induced from the orientation of $\tau$ ) and that of $\tau_{i}$, we may choose an orientation of $v_{i}$ such that the orientation of $\left.\tau\right|_{M_{i}}$ coincides with that of $\tau_{i} \oplus v_{i}$ (thus, we may regard $v_{i}$ as the complex line bundle over $M_{i}$ ). Because $T_{i}$ acts on $v_{i}$, we may choose an orientation of $T_{i}$ such that the $T_{i}$-action preserves the orientation of $v_{i}$. This orientation of $T_{i}$ determines the sign of $\lambda\left(F_{i}\right)$ for $i=1, \ldots, m$. In this way, we have the function

$$
\lambda_{\mathbb{O}}: \mathscr{F}(Q) \rightarrow \mathfrak{t}_{\mathbb{Z}}
$$

In this paper, this is called an omnioriented characteristic function (of $(M, \mathcal{O})$ ).

Remark 2.4. The characteristic function defined in [Wiemeler 2013] may be regarded as the characteristic function $\lambda$ above. On the other hand, the characteristic function defined in [Davis and Januszkiewicz 1991] may be regarded as the characteristic function $\lambda_{\odot}$ above by taking an appropriate omniorientation (see also [Buchstaber and Panov 2002, Section 5.2]).

Let $p \in M^{T}$. We define the subset $I_{p} \subset[m]$ by

$$
I_{p}=\left\{i \in[m] \mid p \in M_{i}\right\} .
$$

By the differentiable slice theorem around $p \in M^{T}$, we have that its cardinality $\left|I_{p}\right|$ equals $n$ for every $p \in M^{T}$. Put $I_{p}=\left\{i_{1}, \ldots, i_{n}\right\}$. Because the $T$-action on $M$ is effective, $\left\{\lambda\left(F_{i_{1}}\right), \ldots, \lambda\left(F_{i_{n}}\right)\right\}$ spans $t_{\mathbb{Z}}^{*} /\{ \pm 1\}$, i.e., the determinant of the induced $(n \times n)$-matrix

$$
\left(\lambda\left(F_{i_{1}}\right) \cdots \lambda\left(F_{i_{n}}\right)\right)
$$

satisfies

$$
\operatorname{det}\left(\lambda\left(F_{i_{1}}\right) \cdots \lambda\left(F_{i_{n}}\right)\right)= \pm 1
$$

Similarly, we have

$$
\operatorname{det}\left(\lambda_{\odot}\left(F_{i_{1}}\right) \cdots \lambda_{\odot}\left(F_{i_{n}}\right)\right)= \pm 1
$$

for each set of $n$ facets such that $\bigcap_{j=1}^{n} F_{i_{j}}=\{p\}$ for some vertex $p \in Q$ (called the facets around a vertex).

Motivated by the above observations, we may abstractly define the characteristic function on a manifold with faces as follows (see [Buchstaber and Panov 2002; Davis and Januszkiewicz 1991] for simple polytopes and [Masuda and Panov 2006; Wiemeler 2013] for manifolds with faces).

Definition 2.5. Let $Q$ be an $n$-dimensional manifold with faces and $\mathscr{F}(Q)$ be the set of its facets. Let $\mathfrak{t}_{\mathbb{Z}}$ be the lattice of the Lie algebra of $T^{n}$ and $\mathfrak{t}_{\mathbb{Z}} /\{ \pm 1\}$ be its quotient 
by $\{ \pm 1\}$. A function $\lambda: \mathscr{F}(Q) \rightarrow \mathfrak{t}_{\mathbb{Z}} /\{ \pm 1\}$ is said to be a characteristic function if $\lambda$ satisfies (2-1) for the facets around every vertex, and a function $\lambda_{\odot}: \mathscr{F}(Q) \rightarrow \mathfrak{t}_{\mathbb{Z}}$ is said to be an omnioriented characteristic function if $\lambda_{\odot}$ satisfies (2-2) for the facets around every vertex.

We denote an $n$-dimensional manifold with faces $Q$ with its characteristic function $\lambda$ (resp. omnioriented characteristic function $\left.\lambda_{\odot}\right)$ by $(Q, \lambda)\left(\operatorname{resp} .\left(Q, \lambda_{\odot}\right)\right)$.

Let $Q_{1}$ and $Q_{2}$ be manifolds with faces, and let $\lambda_{1}$ and $\lambda_{2}$ be their characteristic functions and $\lambda_{\mathrm{O}_{1}}$ and $\lambda_{\mathrm{O}_{2}}$ be their omnioriented characteristic functions, respectively. Assume that $Q_{1} \approx_{c} Q_{2}$, induced by the bijective map $\tilde{f}: \mathscr{P}\left(Q_{1}\right) \rightarrow \mathscr{P}\left(Q_{2}\right)$. Denote its restriction onto the set of facets by

$$
f=\left.\tilde{f}\right|_{\mathscr{F}\left(Q_{1}\right)}: \mathscr{F}\left(Q_{1}\right) \rightarrow \mathscr{F}\left(Q_{2}\right) .
$$

We say that $\left(Q_{1}, \lambda_{1}\right)$ and $\left(Q_{2}, \lambda_{2}\right)$ are combinatorially equivalent if the following diagram commutes:

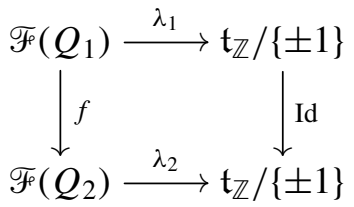

Similarly, $\left(Q_{1}, \lambda_{\Theta_{1}}\right)$ and $\left(Q_{2}, \lambda_{\Theta_{2}}\right)$ are combinatorially equivalent if the following diagram commutes:

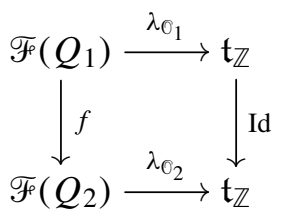

Note that the characteristic function $\lambda$ can be obtained by ignoring signs from the omnioriented characteristic function $\lambda_{\odot}$; we call such a $\lambda$ an induced characteristic function from $\lambda_{\mathscr{\odot}}$. On the other hand, by choosing a sign for each facet, we can obtain an omnioriented characteristic function $\lambda_{\odot}$ from the characteristic function $\lambda$; we call such a $\lambda_{\odot}$ an induced oriented characteristic function from $\lambda$.

Lemma 2.6. If $\left(Q_{1}, \lambda_{\Theta_{1}}\right)$ and $\left(Q_{2}, \lambda_{\Theta_{2}}\right)$ are combinatorially equivalent, then their induced $\left(Q_{1}, \lambda_{1}\right)$ and $\left(Q_{2}, \lambda_{2}\right)$ are also combinatorially equivalent.

If $\left(Q_{1}, \lambda_{1}\right)$ and $\left(Q_{2}, \lambda_{2}\right)$ are combinatorially equivalent, then there are induced omnioriented characteristic functions $\lambda_{\Theta_{1}}$ and $\lambda_{\Theta_{2}}$ such that $\left(Q_{1}, \lambda_{\Theta_{1}}\right)$ and $\left(Q_{2}, \lambda_{\Theta_{2}}\right)$ are combinatorially equivalent.

We now introduce one of the key facts used to prove our main theorem (see [Wiemeler 2013, Theorems 1.3 and 6.1]).

Theorem 2.7 (Wiemeler). Let $M_{1}$ and $M_{2}$ be 6-dimensional simply connected torus manifolds with $H^{\text {odd }}\left(M_{1}\right)=H^{\text {odd }}\left(M_{2}\right)=0$, and let $\left(Q_{1}, \lambda_{1}\right)$ and $\left(Q_{2}, \lambda_{2}\right)$ be 
their orbit spaces with characteristic functions. Then, the following statements are equivalent:

(1) $\left(Q_{1}, \lambda_{1}\right)$ and $\left(Q_{2}, \lambda_{2}\right)$ are combinatorially equivalent.

(2) $M_{1}$ and $M_{2}$ are equivariantly homeomorphic.

(3) $M_{1}$ and $M_{2}$ are equivariantly diffeomorphic.

Therefore, by Corollary 2.3 and Theorem 2.7, to classify all 6-dimensional simply connected torus manifolds with $H^{\text {odd }}(M)=0$, it is enough to classify all $(Q, \lambda)$ 's up to combinatorial equivalence, where $Q$ is a 3-dimensional disk equipped with the structure of a manifold with faces.

\section{Torus graph induced from manifold with faces}

Let $(M, O)$ be an omnioriented locally standard $2 n$-dimensional torus manifold and $\left(Q, \lambda_{\odot}\right)$ be its orbit space with an omnioriented characteristic function. From the one-skeleton of $\left(Q, \lambda_{\odot}\right)$, we can define a labelled graph called a torus graph. One of the key steps in proving the main theorem is to classify all possible torus graphs (see Section 7). We first recall the definition of torus graph given by Maeda et al. [2007].

Let $\Gamma$ be the graph of $Q$. Let $V(\Gamma)$ be its vertices and $E(\Gamma)$ be its oriented edges, i.e., we distinguish two edges $p q$ and $q p$. For $p \in V(\Gamma)$, we denote the set of outgoing edges from $p$ by $E_{p}(\Gamma)$. Because $Q$ is an $n$-dimensional manifold with faces, $\left|E_{p}(\Gamma)\right|=n$ and each edge $e \in E(\Gamma)$ is a connected component of $\bigcap_{i=1}^{n-1} F_{i}$ for some $F_{1}, F_{2}, \ldots, F_{n-1} \in \mathscr{F}(Q)$. Moreover, for a $p \in V(\Gamma)$ which is one of two vertices on $e$, there is another facet $F_{n} \in \mathscr{F}(Q)$ such that $\{p\}$ is a connected component of $\bigcap_{i=1}^{n} F_{i}$. In other words, $F_{n}$ may be regarded as a normal facet of $e \in E(\Gamma)$ on $p \in V(\Gamma)$. Put $\lambda_{\mathscr{O}}\left(F_{i}\right)=a_{i} \in \mathfrak{t}_{\mathbb{Z}} \simeq \mathbb{Z}^{n}$. Then, there exists a unique $\alpha \in \mathfrak{t}_{\mathbb{Z}}^{*}$ such that

$$
\left\langle\alpha, a_{i}\right\rangle=0 \text { for } i=1, \ldots, n-1 \quad \text { and } \quad\left\langle\alpha, a_{n}\right\rangle=+1,
$$

where $\langle\cdot, \cdot\rangle$ represents the pairing of $\mathfrak{t}^{*}$ and $\mathfrak{t}$. Therefore, in this way, we can define a map $\mathscr{A}: E(\Gamma) \rightarrow \mathfrak{t}_{\mathbb{Z}}^{*}$ from the omnioriented characteristic function $\lambda_{\odot}$. This map $\mathscr{A}$ is called an axial function on $\Gamma$. We call the labelled graph $(\Gamma, \mathscr{A})$ a torus graph induced from $\left(Q, \lambda_{\odot}\right)$ (or equivalently $(M, \mathcal{O})$ ). We denote such a torus graph by $\Gamma\left(Q, \lambda_{\odot}\right)$ (or $\left(\Gamma_{M}, A_{M}\right)$ ). We can easily check the following proposition using the definition of torus graph (see also [Maeda et al. 2007]).

Proposition 3.1. Let $(\Gamma, \mathscr{A})$ be a torus graph induced from $\left(Q, \lambda_{\odot}\right)$. Then, $\Gamma$ is an $n$-valent regular graph, i.e., $\left|E_{p}(\Gamma)\right|=n$ for all $p \in V(\Gamma)$, and $(\Gamma, \mathscr{A})$ satisfies the following conditions:

(1) $\mathscr{A}(e)= \pm \mathscr{A}(\bar{e})$, where $\bar{e}$ is the orientation-reversed edge of $e$. 
(2) $\left\{\mathscr{A}(e) \mid e \in E_{p}(\Gamma)\right\}$ spans $\mathfrak{t}_{\mathbb{Z}}^{*}$ for all vertices $p \in V(\Gamma)$.

(3) There is a bijection $\nabla_{p q}: E_{p}(\Gamma) \rightarrow E_{q}(\Gamma)$ for all edges whose initial vertex is $p$ and terminal vertex is $q$ such that

(a) $\nabla_{\bar{e}}=\nabla_{e}^{-1}$,

(b) $\nabla_{e}(e)=\bar{e}$,

(c) $\mathscr{A}\left(\nabla_{p q}(e)\right)-\mathscr{A}(e) \equiv 0 \bmod \mathscr{A}(p q)$ for all $e \in E_{p}(\Gamma)$.

We call $\nabla=\left\{\nabla_{e} \mid e \in E(\Gamma)\right\}$ a connection on $(\Gamma, \mathscr{A})$.

Remark 3.2. The original definition of torus graph (induced from an omnioriented torus manifold) uses tangential representations; see [Masuda and Panov 2006; Maeda et al. 2007]. The definition of torus graph given above is essentially the same as the original definition.

In [Maeda et al. 2007], motivated by the GKM graph introduced by Guillemin and Zara [2001], an $n$-valent graph $\Gamma$ with a label $\mathscr{A}: E(\Gamma) \rightarrow \mathfrak{t}_{\mathbb{Z}}^{*}$ which satisfies the three conditions in Proposition 3.1 is called an (abstract) torus graph (i.e., there might be no geometric objects which define $(\Gamma, \mathscr{A})$ ).

We next define the equivalence relation between two torus graphs. We call the map $f: \Gamma_{1}=\left(V\left(\Gamma_{1}\right), E\left(\Gamma_{1}\right)\right) \rightarrow \Gamma_{2}=\left(V\left(\Gamma_{2}\right), E\left(\Gamma_{2}\right)\right)$ a graph isomorphism if the restricted maps $\left.f\right|_{V}: V\left(\Gamma_{1}\right) \rightarrow V\left(\Gamma_{2}\right)$ and $\left.f\right|_{E}: E\left(\Gamma_{1}\right) \rightarrow E\left(\Gamma_{2}\right)$ are bijective and the following diagram commutes:

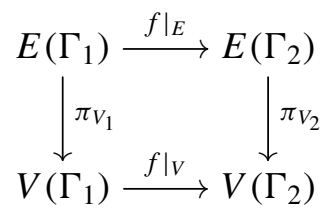

Here the map $\pi_{V}: E(\Gamma) \rightarrow V(\Gamma)$ is the projection onto the initial vertex, i.e., $\pi_{V}(p q)=p$. In other words, the bijection $\left.f\right|_{V}$ preserves the edges. Now we may define the equivalence relation.

Definition 3.3. Let $\left(\Gamma_{1}, \mathscr{A}_{1}\right)$ and $\left(\Gamma_{2}, \mathscr{A}_{2}\right)$ be torus graphs. We say $\left(\Gamma_{1}, \mathscr{A}_{1}\right)$ and $\left(\Gamma_{2}, \mathscr{A}_{2}\right)$ are equivalent if there is a graph isomorphism $f: \Gamma_{1} \rightarrow \Gamma_{2}$ such that the following diagram commutes:

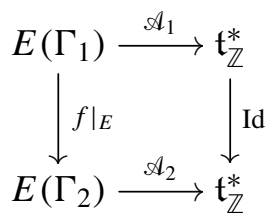


Assume $(\Gamma, \mathscr{A})=\Gamma\left(Q, \lambda_{\mathscr{O}}\right)$. Let $\mathscr{P}_{k}(\Gamma, \mathscr{A})$ be the set of $k$-valent torus subgraphs in $(\Gamma, \mathscr{A})$, i.e., $k$-valent subgraphs in $\Gamma$ closed under the connection $\nabla$, where $-1 \leq k \leq n$ and we define $\mathscr{P}_{-1}(\Gamma, \mathscr{A})=\{\varnothing\}$. Then, the set

$$
\mathscr{P}(\Gamma, \mathscr{A})=\bigcup_{k=-1}^{n} \mathscr{P}_{k}(\Gamma, \mathscr{A})
$$

admits the structure of a simplicial poset by inclusion (see [Maeda et al. 2007]). We denote this structure by $(\mathscr{P}(\Gamma, \mathscr{A}), \preceq)$. Let $\mathscr{P}(Q)$ be the face poset of $Q$ (see Section 2B) and $\mathscr{P}_{k}(Q)$ be the set of all $k$-dimensional faces, where $-1 \leq k \leq n$ and $\mathscr{P}_{-1}(Q)=\{\varnothing\}$. Then, each element of $\mathscr{P}_{k}(\Gamma, \mathscr{A})$ is nothing but the graph of an element in $\mathscr{P}_{k}(Q)$. This implies that the poset $(\mathscr{P}(\Gamma, \mathscr{A}), \preceq)$ is equivalent to the poset $(\mathscr{P}(Q), \preceq)$. Therefore, we have the following lemma.

Lemma 3.4. The following two statements are equivalent:

(1) Two manifolds with faces with omnioriented characteristic functions $\left(Q_{1}, \lambda_{\Theta_{1}}\right)$ and $\left(Q_{2}, \lambda_{\mathrm{O}_{2}}\right)$ are combinatorially equivalent.

(2) Their induced torus graphs $\Gamma\left(Q_{1}, \lambda_{\Theta_{1}}\right)$ and $\Gamma\left(Q_{2}, \lambda_{\Theta_{2}}\right)$ are equivalent.

By Lemma 2.6, Theorem 2.7 and Lemma 3.4, we have the following corollary.

Corollary 3.5. Let $\left(M_{1}, T\right)$ and $\left(M_{2}, T\right)$ be 6-dimensional simply connected torus manifolds with vanishing odd-degree cohomology. Then, the following statements are equivalent:

(1) $\left(M_{1}, T\right)$ and $\left(M_{2}, T\right)$ are equivariantly diffeomorphic.

(2) Their orbit spaces, i.e., 3-dimensional disks with the structures of manifolds with faces, with characteristic functions $\left(M_{1} / T, \lambda_{1}\right)$ and $\left(M_{2} / T, \lambda_{2}\right)$ are combinatorially equivalent.

(3) There are omnioriented characteristic functions $\lambda_{\Theta_{1}}$ and $\lambda_{\Theta_{2}}$ such that their induced 3-valent torus graphs $\Gamma\left(M_{1} / T, \lambda_{\Theta_{1}}\right)$ and $\Gamma\left(M_{2} / T, \lambda_{\mathscr{O}_{2}}\right)$ are equivalent.

Therefore, to prove our main theorem (Theorem 7.1), it is enough to classify all 3 -valent torus graphs $(\Gamma, \mathscr{A})$, induced from $(M, \mathcal{O})$, up to equivalence.

\section{Basic 6-dimensional torus manifolds}

Let $(M, T)$ be a simply connected 6-dimensional torus manifold with $H^{\text {odd }}(M)=0$, and let $\left(\Gamma_{M}, \mathscr{A}_{M}\right)(=(\Gamma, \mathscr{A}))$ be its torus graph induced by some omniorientation. As a preliminary to proving the main theorem (Theorem 7.1), in this section we introduce some of the basic torus graphs $(\Gamma, \mathscr{A})$ and their corresponding 6dimensional torus manifolds $(M, T)$. 


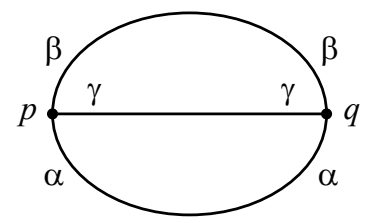

Figure 1. The torus graph $\left(\Gamma_{\mathrm{sp}}, \mathscr{A}_{\alpha, \beta, \gamma}\right)$, where $\alpha, \beta, \gamma \in \mathfrak{t}_{\mathbb{Z}}^{*} \simeq \mathbb{Z}^{3}$ are a $\mathbb{Z}$-basis.

4A. 6-sphere. Because the induced torus graphs from $(M, T)$ are 3-valent, if there is a 3-multiple edge, i.e., three edges that are incident to the same two vertices, then it follows from Proposition 3.1 that such a torus graph must be the torus graph in Figure 1 , denoted $\left(\Gamma_{\mathrm{sp}}, \mathcal{A}_{\alpha, \beta, \gamma}\right)$.

Put $\alpha=k_{11} e_{1}+k_{12} e_{2}+k_{13} e_{3}, \beta=k_{21} e_{1}+k_{22} e_{2}+k_{23} e_{3}$ and $\gamma=k_{31} e_{1}+k_{32} e_{2}+$ $k_{33} e_{3}$, using the standard basis $e_{1}, e_{2}, e_{3}$ in $\mathfrak{t}_{\mathbb{Z}}^{*} \simeq \mathbb{Z}^{3}$. Then, we have

$$
\operatorname{det}\left(\begin{array}{lll}
k_{11} & k_{12} & k_{13} \\
k_{21} & k_{22} & k_{23} \\
k_{31} & k_{32} & k_{33}
\end{array}\right)= \pm 1 .
$$

Let $S^{6} \subset \mathbb{C}^{3} \oplus \mathbb{R}$ be the unit sphere, i.e., the set $\left(z_{1}, z_{2}, z_{3}, r\right) \in \mathbb{C}^{3} \oplus \mathbb{R}$ such that $\left|z_{1}\right|^{2}+\left|z_{2}\right|^{2}+\left|z_{3}\right|^{2}+r^{2}=1$. Define the $T^{3}$-action on the first three complex coordinates in $S^{6}$ by

$$
\left(t_{1}, t_{2}, t_{3}\right)\left(z_{1}, z_{2}, z_{3}, r\right) \mapsto\left(\rho_{1}(t) z_{1}, \rho_{2}(t) z_{2}, \rho_{3}(t) z_{3}, r\right),
$$

where $t=\left(t_{1}, t_{2}, t_{3}\right) \in T$ and $\rho_{i}: T \rightarrow S^{1}, i=1,2,3$, is a 1 -dimensional complex representation defined by

$$
\rho_{i}\left(t_{1}, t_{2}, t_{3}\right)=t_{1}^{k_{i 1}} t_{2}^{k_{i 2}} t_{3}^{k_{i 3}} .
$$

Then, by choosing an appropriate omniorientation on $S^{6}$, we have that its induced torus graph is equivalent to $\left(\Gamma_{\mathrm{sp}}, \mathcal{A}_{\alpha, \beta, \gamma}\right)$. Therefore, using Corollary 3.5 , we have the following lemma.

Lemma 4.1. Let $(M, O)$ be an omnioriented 6-dimensional simply connected torus manifold with $H^{\text {odd }}(M)=0$. If its induced torus graph is $\left(\Gamma_{\mathrm{sp}}, \mathcal{A}_{\alpha, \beta, \gamma}\right)$, then $(M, T)$ is equivariantly diffeomorphic to one of $\left(S^{6}, T\right)$ defined by (4-2).

4B. $S^{4}$-bundles over $S^{2}$. Assume that a 3 -valent torus graph $(\Gamma, \mathscr{A})$ does not have 3-multiple edges but does have multiple edges, i.e., two edges that are incident to the same two vertices. In this section, we classify the easiest case of such torus graphs.

Because $\Gamma$ is a one-skeleton of a 3-dimensional manifold with faces $Q$, we have $|V(\Gamma)| \geq 4$. Assume that $|V(\Gamma)|=4$. Then, we can easily check that such a torus 


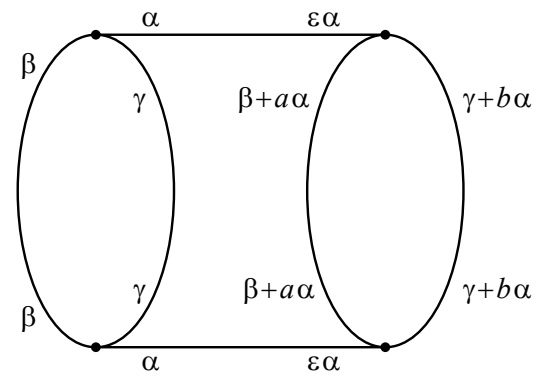

Figure 2. The torus graph $\left(\Gamma_{S}, \mathscr{A}_{S}\right)=\left(\Gamma_{S}, \mathscr{A}_{\alpha, \beta, \gamma}^{\epsilon, a, b}\right)$, where $\epsilon= \pm 1$, $a, b \in \mathbb{Z}$, and $\alpha, \beta, \gamma \in \mathfrak{t}_{\mathbb{Z}}^{*}$ are a $\mathbb{Z}$-basis of $\mathfrak{t}_{\mathbb{Z}}^{*}$.

manifold is the one-skeleton of the 3 -simplex (see Figure 3 in Section 4C) or the graph drawn in Figure 2, say $\Gamma_{S}$. It is well known that the torus manifold whose torus graph is the one-skeleton of the 3-simplex is equivariantly diffeomorphic to the complex projective space with some $T$-action (see, e.g., [Davis and Januszkiewicz 1991], and see also Figure 3 in Section 4C). So, we only study the torus manifold which induces the graph $\Gamma_{S}$. Because $Q$ is homeomorphic to $D^{3}$, we may regard a $Q$ whose one-skeleton is $\Gamma_{S}$ as the product $D^{2} \times I$, where $D^{2}$ is the 2-dimensional disk and $I$ is the interval. By considering all functions on facets of $Q$ which satisfy (2-2), we can classify all omnioriented characteristic functions $\lambda_{0}$ on $Q$. Then, in the same way we induced the axial function $\mathscr{A}_{S}$ from $\left(Q, \lambda_{\odot}\right)$ in Section 3 , we can obtain all possible axial functions on $\Gamma_{S}$, as shown in Figure 2.

The torus graph $\left(\Gamma_{S}, \mathscr{A}_{S}\right)$ in Figure 2 can be induced from an $S^{4}$-bundle over $S^{2}$ as follows. First, by choosing $\epsilon= \pm 1$, we may define two free $T^{1}$-actions on $S^{3} \subset \mathbb{C}^{2}$ :

$$
(w, z) \mapsto\left(t^{-1} w, t^{\epsilon} z\right)
$$

We denote $S^{3}$ with the above $T^{1}$-action by $S_{\epsilon}^{3}$. Note that $S_{\epsilon}^{3} / T^{1}$ is diffeomorphic to the 2-sphere $S^{2}$, and a complex line bundle over $S^{2}$ can be denoted by

$$
S_{\epsilon}^{3} \times_{T^{1}} \mathbb{C}_{k}
$$

where $\mathbb{C}_{k}$ is the complex 1-dimensional $T^{1}$-representation space by $k$-times rotation for some $k \in \mathbb{Z}$. Let $S_{\epsilon}^{3} \times_{T^{1}} \mathbb{R}$ be the trivial real line bundle over $S^{2}$. Take the unit sphere bundle of the following Whitney sum of three vector bundles for $a, b \in \mathbb{Z}$ :

$$
S_{\epsilon}^{3} \times_{T^{1}}\left(\mathbb{C}_{a} \oplus \mathbb{C}_{b} \oplus \mathbb{R}\right) .
$$

Then, we obtain the $S^{4}$-bundle over $S^{2}$ denoted by

$$
M(\epsilon, a, b)=S_{\epsilon}^{3} \times_{S^{1}} S\left(\mathbb{C}_{a} \oplus \mathbb{C}_{b} \oplus \mathbb{R}\right),
$$


for $\epsilon= \pm 1, a, b \in \mathbb{Z}$. Namely, we can identify elements in $M(\epsilon, a, b)$ by

$$
[(w, z),(x, y, r)]=\left[\left(t^{-1} w, t^{\epsilon} z\right),\left(t^{a} x, t^{b} y, r\right)\right]
$$

for any $t \in T^{1}$ such that $|w|^{2}+|z|^{2}=1$ and $|x|^{2}+|y|^{2}+r^{2}=1$. Define a $T^{3}$-action on $M(\epsilon, a, b)$ by

$$
[(w, z),(x, y, r)] \mapsto\left[\left(t_{1} w, z\right),\left(t_{2} x, t_{3} y, r\right)\right],
$$

where $\left(t_{1}, t_{2}, t_{3}\right) \in T^{3}$. Fix an omniorientation on $M(\epsilon, a, b)$ by the induced orientations from $S_{\epsilon}^{3} \times S^{4} \subset \mathbb{C}^{2} \times(\mathbb{C} \oplus \mathbb{C} \oplus \mathbb{R})$. Then, considering the tangential representations around each fixed point, it is easy to check that the induced torus graph is $\left(\Gamma_{S}, \mathcal{A}_{e_{1}, e_{2}, e_{3}}^{\epsilon, a, b}\right)$, where $e_{1}, e_{2}, e_{3}$ are the standard basis of $\mathfrak{t}_{\mathbb{Z}} \simeq \mathbb{Z}^{3}$. Therefore, by taking the appropriate automorphism of $T^{3}$, we can construct each torus graph $\left(\Gamma_{S}, \mathscr{A}_{S}\right)$ in Figure 2 from $M(\epsilon, a, b)$. Note that if $\epsilon=-1$ and $a=b$, then this is nothing but one of the torus manifolds which appeared in the classifications of torus manifolds with codimension-1 extended actions in [Kuroki 2011].

By the argument above and Corollary 3.5, we have the following lemma.

Lemma 4.2. Let $(M, \mathcal{O})$ be an omnioriented 6-dimensional simply connected torus manifold with $H^{\text {odd }}(M)=0$. If its induced torus graph has four vertices, then $(M, T)$ is equivariantly diffeomorphic to one of the following:

(1) $\mathbb{C} P^{3}$ with the standard $T^{3}$-action up to automorphism of $T^{3}$;

(2) $M(\epsilon, a, b)$ for some $\epsilon= \pm 1$ and $a, b \in \mathbb{Z}$.

4C. 6-dimensional quasitoric manifolds. Assume that there are no multiple edges in a 3 -valent torus graph $(\Gamma, \mathscr{A})$, i.e., there are no two edges that are incident to the same two vertices. A graph $\Gamma$ is called simple if $\Gamma$ does not have both multiple edges and loops. In this section and in Section 5, we study simple torus graphs which can be realized as the one-skeleton of a manifold with faces homeomorphic to $D^{3}$.

The typical example of such torus manifolds whose torus graphs are simple is a quasitoric manifold (introduced by Davis and Januszkiewicz [1991]; see also [Buchstaber and Panov 2002]). A quasitoric manifold is defined by a torus manifold whose orbit space is a simple convex polytope, i.e., a convex polytope admitting the structure of a manifold with faces. For example, the complex projective space $\mathbb{C} P^{n}$ with the standard $T^{n}$-action is the quasitoric manifold whose orbit space is the $n$-dimensional simplex. Figure 3 shows the torus graph induced from $\left(\mathbb{C} P^{3}, \mathscr{O}_{\mathbb{C}}\right)$, i.e., the omniorientation $\mathbb{O}_{\mathbb{C}}$ induced from the standard complex structure on $\mathbb{C} P^{3}$ and the standard $T$-action on $\mathbb{C} P^{3}$.

We next characterize when torus graphs are induced from simple convex polytopes, i.e., induced from quasitoric manifolds. The Steinitz theorem (see [Ziegler 


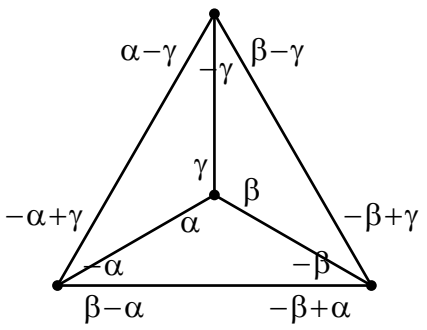

Figure 3. The torus graph induced from $\left(\mathbb{C} P^{3}, \mathbb{O}_{\mathbb{C}}\right)$.

1995, Chapter 4]) tells us that a graph $\Gamma$ is the one-skeleton of a 3-dimensional convex polytope if and only if $\Gamma$ is a simple, planar and 3-connected graph, where $\Gamma$ is called a 3-connected graph if it remains connected whenever fewer than three vertices are removed. It easily follows from the Steinitz theorem that we have the following lemma.

Lemma 4.3. Let $Q$ be a manifold with faces and $\Gamma$ be its graph. Assume that $Q$ is homeomorphic to the 3-disk $D^{3}$ and there are no multiple edges. Then, the following statements are equivalent:

(1) $Q$ is combinatorially equivalent to a 3-dimensional simple convex polytope $P$.

(2) $\Gamma$ is a 3-connected graph.

Combining this result with Corollary 3.5, we have the following fact.

Lemma 4.4. Let $(M, 0)$ be an omnioriented 6-dimensional simply connected torus manifold with $H^{\text {odd }}(M)=0$. Then, the following statements are equivalent:

(1) $(M, T)$ is equivariantly diffeomorphic to a quasitoric manifold.

(2) Its induced torus graph $\Gamma$ is a 3-connected graph with no multiple edges.

\section{Connected sum of torus graphs and other 6-dimensional torus manifolds}

By the arguments in Section 4, only the following case remains: the simply connected 6-dimensional torus manifolds with $H^{\text {odd }}(M)=0$ whose induced torus graphs are simple but not 3-connected. Such torus manifolds can be constructed using the connected sum of "oriented" torus graphs. The purpose of this section is to introduce oriented torus graphs and their connected sum (see also [Darby 2015]).

We first recall the equivariant connected sum of torus manifolds. Let $M_{1}, M_{2}$ be $2 n$-dimensional torus manifolds and $p \in M_{1}^{T}, q \in M_{2}^{T}$ be fixed points. Using the slice theorem, we may take $T$-invariant open neighborhoods $U_{1} \subset M_{1}$ of $p$ and $U_{2} \subset M_{2}$ of $q$. Assume that $U_{1}$ and $U_{2}$ are equivariantly diffeomorphic. Then, $U_{1} \backslash\{p\}$ and $U_{2} \backslash\{q\}$ are equivariantly diffeomorphic to $S^{2 n-1} \times I$, where $S^{2 n-1} \subset \mathbb{C}^{n}$ with some effective $T^{n}$-action and $I=(-\epsilon, \epsilon)$ with the trivial $T^{n}$-action for some 
$\epsilon>0$. We glue these two neighborhoods by $\varphi$ defined by the identity on $S^{2 n-1}$ and the map $r \mapsto-r$ on $I$ for $r \in I$. Namely, we can glue $M_{1} \backslash\{p\}$ and $M_{2} \backslash\{q\}$ by the identification

(5-1) $M_{1} \backslash\{p\} \supset U_{1} \backslash\{p\} \stackrel{\simeq}{\longrightarrow} S^{2 n-1} \times I \stackrel{\varphi}{\longrightarrow} S^{2 n-1} \times I \stackrel{\simeq}{\longrightarrow} U_{2} \backslash\{q\} \subset M_{2} \backslash\{q\}$.

The $T^{n}$-manifold obtained in this way is denoted by $M_{1} \# M_{2}$ or $M_{1} \#(p, q) M_{2}$ (if we emphasize fixed points $p \in M_{1}^{T}$ and $q \in M_{2}^{T}$ ). Because each torus manifold has more than two fixed points, $M_{1} \# M_{2}$ is again a torus manifold. We call this operation the equivariant connected sum.

Lemma 5.1. If two torus manifolds $M_{1}$ and $M_{2}$ are simply connected and $H^{\text {odd }}\left(M_{1}\right)$ $=H^{\text {odd }}\left(M_{2}\right)=0$, then $M_{1} \# M_{2}$ is also simply connected and $H^{\text {odd }}\left(M_{1} \# M_{2}\right)=0$.

Proof. It is easy to check the statement using van Kampen's theorem and the Mayer-Vietoris exact sequence.

Assume that $\left(M_{1}, O_{1}\right)$ and $\left(M_{2}, O_{2}\right)$ are 6-dimensional omnioriented simply connected torus manifolds with $H^{\text {odd }}\left(M_{1}\right)=H^{\text {odd }}\left(M_{2}\right)=0$. Let $\left(\Gamma_{1}, \mathscr{A}_{1}\right)$ and $\left(\Gamma_{2}, \mathscr{A}_{2}\right)$ be their induced 3-valent torus graphs. Assume that we can glue $p \in M_{1}^{T}$ and $q \in M_{2}^{T}$ by the connected sum. Then, by considering the restriction of $\varphi$ in (5-1) onto $S^{2 n-1} \subset \mathbb{C}^{n}$, i.e., the identity map, the axial functions around $p \in V\left(\Gamma_{1}\right)$ and $q \in V\left(\Gamma_{2}\right)$ must satisfy

$$
\left\{\mathscr{A}_{1}(e) \mid e \in E_{p}\left(\Gamma_{1}\right)\right\}=\left\{\mathscr{A}_{2}(e) \mid e \in E_{q}\left(\Gamma_{2}\right)\right\} .
$$

However, at this stage, the torus graphs $\left(\Gamma_{1}, \mathscr{A}_{1}\right)$ and $\left(\Gamma_{2}, \mathscr{A}_{2}\right)$ do not contain information about the orientations of $M_{1}$ and $M_{2}$. To do the connected sum, we need the orientations around $p \in M_{1}^{T}$ and $q \in M_{2}^{T}$. To encode the orientations around fixed points, we need the following notion.

Definition 5.2. Let $(\Gamma, \mathscr{A})$ be a torus graph. We call a triple $(\Gamma, \mathscr{A}, \sigma)$ with a map $\sigma: V(\Gamma) \rightarrow\{+1,-1\}$ an oriented torus graph if $\sigma$ satisfies the following condition for all $e \in E(\Gamma)$ :

$$
\sigma\left(\pi_{V}(e)\right) \mathscr{A}(e)=-\sigma\left(\pi_{V}(\bar{e})\right) \mathscr{A}(\bar{e}),
$$

where $\pi_{V}(e) \in V(\Gamma)$ is the initial vertex of $e \in E(\Gamma)$, i.e., for $e=p q, \pi_{V}(e)=p$ and $\pi_{V}(\bar{e})=q$. We call such a map $\sigma$ an orientation of $(\Gamma, \mathscr{A})$.

Remark 5.3. Let $(M, \mathcal{O})$ be an omnioriented torus manifold. The oriented torus graph $(\Gamma, \mathcal{A}, \sigma)$ of $(M, \mathcal{O})$ is defined as follows. Let $p \in M^{T}$. Then, there exist exactly $n$ characteristic submanifolds $M_{1}, \ldots, M_{n}$ such that $p$ is a connected component of $\bigcap_{i=1}^{n} M_{i}$. Now the fixed orientations of $M_{1}, \ldots, M_{n}$ determine the decomposition of the tangential representation; i.e., $\psi_{p}: T_{p} M \stackrel{\sim}{\rightarrow} V\left(\alpha_{1}\right) \oplus \cdots \oplus V\left(\alpha_{n}\right)$ is determined by fixing the orientations of $M_{1}, \ldots, M_{n}$. On the other hand, the 
orientation of $M$ determines the orientation of $T_{p} M$. So, we define the map $\sigma: V(\Gamma)=M^{T} \rightarrow\{+1,-1\}$ by

$$
\sigma(p)= \begin{cases}+1 & \text { if } \psi_{p} \text { preserves the orientations, } \\ -1 & \text { if } \psi_{p} \text { reverses the orientations. }\end{cases}
$$

Let $\left(\Gamma_{1}, \mathscr{A}_{1}, \sigma_{1}\right)$ and $\left(\Gamma_{2}, \mathscr{A}_{2}, \sigma_{2}\right)$ be the induced oriented torus graphs from $\left(M_{1}, O_{1}\right)$ and $\left(M_{2}, O_{2}\right)$. If we can glue $p \in M_{1}^{T}$ and $q \in M_{2}^{T}$ by the connected sum, then both (5-2) and

$$
\sigma_{1}(p) \neq \sigma_{2}(q)
$$

hold ((5-3) corresponds to the fact that the orientations on $T_{p} M_{1}$ and $T_{q} M_{2}$ are different). The induced (oriented) torus graph by $M_{1} \#_{(p, q)} M_{2}$ is nothing but the one-skeleton of the connected sum $Q_{1} \#_{(p, q)} Q_{2}$ of manifolds with faces, where $Q_{i}$ is the orbit space of $M_{i}, i=1,2$ (see [Izmest'ev 2001, Definition 3; Kuroki 2010, Section 3.1] for details about the connected sum of polytopes). Therefore, conversely, if $p \in V\left(\Gamma_{1}\right)$ and $q \in V\left(\Gamma_{2}\right)$ satisfy (5-2) and (5-3), then we can do the connected sum of (oriented) torus graphs between $\left(\Gamma_{1}, \mathscr{A}_{1}, \sigma_{1}\right)$ and $\left(\Gamma_{2}, \mathscr{A}_{2}, \sigma_{2}\right)$, say $(\Gamma, \mathscr{A}, \sigma)=\left(\Gamma_{1}, \mathscr{A}_{1}, \sigma_{1}\right) \#\left(\Gamma_{2}, \mathscr{A}_{2}, \sigma_{2}\right)$ or $\left(\Gamma_{1}, \mathscr{A}_{1}, \sigma_{1}\right) \#_{(p, q)}\left(\Gamma_{2}, \mathscr{A}_{2}, \sigma_{2}\right)$ (if we emphasize the vertices $p \in V\left(\Gamma_{1}\right)$ and $\left.q \in V\left(\Gamma_{2}\right)\right)$. More precisely, $(\Gamma, \mathscr{A}, \sigma)=$ $\left(\Gamma_{1}, \mathscr{A}_{1}, \sigma_{1}\right) \#\left(\Gamma_{2}, \mathscr{A}_{2}, \sigma_{2}\right)$ is defined as follows (see Figure 4).

(1) $V(\Gamma)=V\left(\Gamma_{1}\right) \backslash\{p\} \sqcup V\left(\Gamma_{2}\right) \backslash\{q\}$.

(2) $E(\Gamma)$ is given by

$\left(E\left(\Gamma_{1}\right) \backslash\left\{p p_{1}, p p_{2}, p p_{3}\right\}\right) \sqcup\left(E\left(\Gamma_{2}\right) \backslash\left\{q q_{1}, q q_{2}, q q_{3}\right\}\right) \sqcup\left\{p_{1} q_{1}, p_{2} q_{2}, p_{3} q_{3}\right\}$, where $\mathscr{A}_{1}\left(p p_{i}\right)=\mathscr{A}_{2}\left(q q_{i}\right)$ for $i=1,2,3$.

(3) $\mathscr{A}: E(\Gamma) \rightarrow\left(\mathfrak{t}_{\mathbb{Z}}^{3}\right)^{*}$ is defined by $\mathscr{A}(e)=\mathscr{A}_{1}(e)$ and $\mathscr{A}(f)=\mathscr{A}_{2}(f)$ for $e$ in $E\left(\Gamma_{1}\right) \backslash\left\{p p_{1}, p p_{2}, p p_{3}\right\}$ and $f$ in $E\left(\Gamma_{2}\right) \backslash\left\{q q_{1}, q q_{2}, q q_{3}\right\}$, and $\mathscr{A}\left(p_{i} q_{i}\right)=$ $\mathscr{A}_{1}\left(p_{i} p\right)$ and $\mathscr{A}\left(q_{i} p_{i}\right)=\mathscr{A}_{2}\left(q_{i} q\right)$.

(4) $\sigma: V(\Gamma) \rightarrow\{+1,-1\}$ is defined by $\sigma(r)=\sigma_{1}(r)$ for $r \in V\left(\Gamma_{1}\right) \backslash\{p\}$ and $\sigma\left(r^{\prime}\right)=\sigma_{2}\left(r^{\prime}\right)$ for $r^{\prime} \in V\left(\Gamma_{2}\right) \backslash\{q\}$.
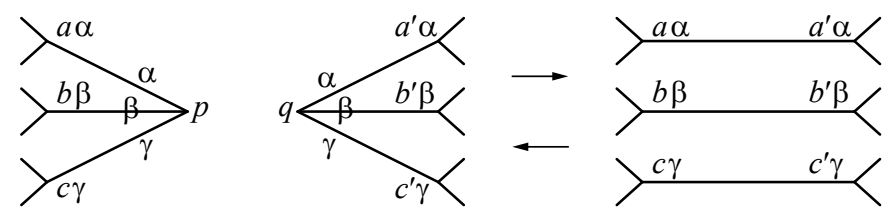

Figure 4. The local figure of the connected sum $\#_{(p, q)}$ of a torus manifold (left to right) and its inverse $\#_{(p, q)}^{-1}$ (right to left), where $\sigma_{1}(p) \neq \sigma_{2}(q)$. Here, $\alpha, \beta, \gamma$ are a $\mathbb{Z}$-basis of $\left(\mathfrak{t}_{\mathbb{Z}}^{3}\right)^{*}$ and $a, a^{\prime}, b, b^{\prime}, c, c^{\prime}= \pm 1$. 

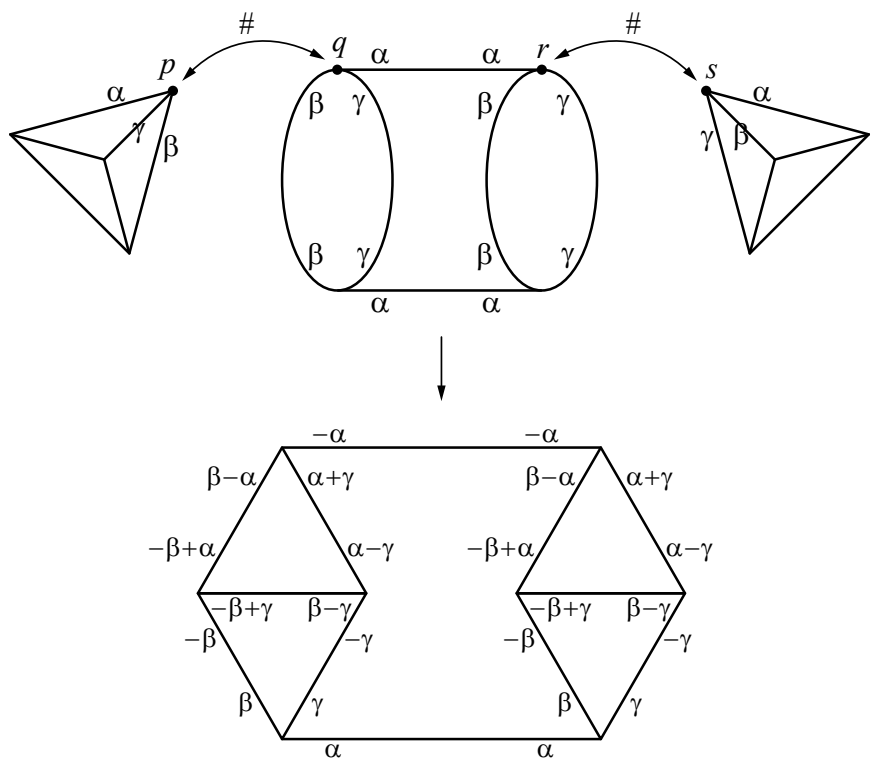

Figure 5. The torus graph (with appropriate orientations, e.g, $\sigma(p)=+1, \sigma(q)=-1, \sigma(r)=+1, \sigma(s)=-1)$ induced from $\mathbb{C} P^{3} \#\left(S^{2} \times S^{4}\right) \# \overline{\mathbb{C} P^{3}}$.

Then, we can easily check that $(\Gamma, \mathcal{A}, \sigma)$ is an oriented torus graph. Using Corollary 3.5 and the arguments above, we have the following lemma.

Lemma 5.4. Let $M_{1}$ and $M_{2}$ be 6-dimensional simply connected torus manifolds with $H^{\text {odd }}\left(M_{1}\right)=H^{\text {odd }}\left(M_{2}\right)=0$, and let $\left(\Gamma_{1}, A_{1}, \sigma_{1}\right)$ and $\left(\Gamma_{2}, \mathscr{A}_{2}, \sigma_{2}\right)$ be their respective induced oriented torus graphs from some omniorientations. If $(\Gamma, \mathcal{A}, \sigma)=\left(\Gamma_{1}, \mathscr{A}_{1}, \sigma_{1}\right) \#_{(p, q)}\left(\Gamma_{2}, \mathscr{A}_{2}, \sigma_{2}\right)$, then $(\Gamma, \mathcal{A}, \sigma)$ is the oriented torus graph induced from $M=M_{1} \#_{(p, q)} M_{2}$ with some omniorientation.

Using the connected sum, we can construct the torus manifolds which do not appear in Section 4. One such example is

$$
\mathbb{C} P^{3} \#\left(S^{2} \times S^{4}\right) \# \overline{\mathbb{C} P^{3}},
$$

where $\overline{\mathbb{C} P^{3}}$ is the reversed orientation of $\mathbb{C} P^{3}$. Figure 5 shows the torus graph induced from $\mathbb{C} P^{3} \#\left(S^{2} \times S^{4}\right) \# \overline{\mathbb{C} P^{3}}$ (see the axial functions in Figures 2 and 3 for details). We can easily check that this graph is 3-valent, simple and planar but not 3-connected; therefore, by Lemma 4.4, this manifold is not a quasitoric manifold.

\section{Some combinatorial lemmas}

To prove the main theorem (Theorem 7.1), we need the following two lemmas. 


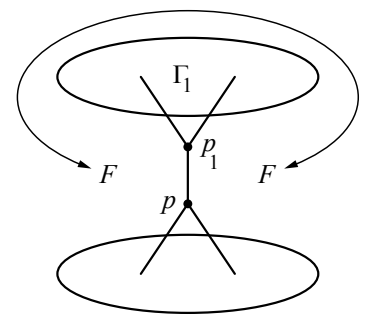

Figure 6. The figure explained in the proof of Lemma 6.1. The facet $F$ has a self-intersection on the edge $p_{1} p$.

Lemma 6.1. Let $Q$ be a 3-dimensional manifold with faces which is homeomorphic to $D^{3}$ and $\Gamma$ be its graph. Then, $\Gamma \backslash\{p\}$ is connected for all vertices $p \in V(\Gamma)$.

Proof. Because $Q$ is homeomorphic to the 3-disk $D^{3}, \Gamma$ may be regarded as a planar graph by the stereographic projection of $\partial Q=S^{2}$. Assume $\Gamma \backslash\{p\}$ is not connected. Because $Q$ is a 3-dimensional manifold with faces, there are exactly three outgoing edges from $p$, say $p p_{1}, p p_{2}$ and $p p_{3}$. Therefore, we may assume that there exists a connected component $\Gamma_{1}$ in $\Gamma \backslash\{p\}$ such that $p_{1} \in V\left(\Gamma_{1}\right)$ but $p_{2}, p_{3} \notin V\left(\Gamma_{1}\right)$ (see Figure 6). Since $\Gamma_{1}$ is also a planar 3-valent graph except on the vertex $p_{1}$ (because $p \notin V\left(\Gamma_{1}\right)$ ), there is a 2-valent subgraph in $\Gamma_{1}$, say $\partial \Gamma_{1}$, such that $\partial \Gamma_{1}$ splits $\partial Q=S^{2}$ into two connected components $H_{+}$and $H_{-}$, where $\Gamma_{1} \backslash \partial \Gamma_{1} \subset H_{+} \backslash \partial \Gamma_{1}$ but $\Gamma_{1} \not \subset H_{-}$. This implies that there is a facet $F$ in $Q$ such that $\partial F$ contains $\partial \Gamma_{1}$ and $p_{1} p$. However, in this case, $p_{1} p$ must be a self-intersection edge of $F$ (see Figure 6). This contradicts that $Q$ is a manifold with faces.

By Lemma 6.1, if $\Gamma$ is not 3-connected, then there are two vertices $p, q \in V(\Gamma)$ such that $\Gamma \backslash\{p, q\}$ is not connected but both $\Gamma \backslash\{p\}$ and $\Gamma \backslash\{q\}$ are connected. More precisely, we have the following lemma.

Lemma 6.2. Let $Q$ be a 3-dimensional manifold with faces which is homeomorphic to $D^{3}$ and $\Gamma$ be its graph. Assume that there are two vertices $p, q \in V(\Gamma)$ such that $\{p, q\} \not \subset V(F)$ for any facets $F$, i.e, $p$ and $q$ are not on the same facet $F$. Then, $\Gamma \backslash\{p, q\}$ is connected.

Proof. Assume that $p$ and $q$ are not on the same facet of $Q$. Because $Q$ is a manifold with faces, there are mutually distinct facets $F_{1}, \ldots, F_{6}$ such that $\{p\}$ is a component of $F_{1} \cap F_{2} \cap F_{3}$ and $\{q\}$ is a component of $F_{4} \cap F_{5} \cap F_{6}$, and we can take vertices $p_{1}, p_{2}, p_{3}$ and $q_{1}, q_{2}, q_{3}$ such that $p p_{i}$ and $q q_{i}$ are all outgoing edges from $p$ and $q$ for $i=1,2,3$. Take two vertices $r$ and $s$ from $\Gamma \backslash\{p, q\}$. By Lemma 6.1, $\Gamma \backslash\{q\}$ is connected. So there is a path $\gamma$ from $r$ to $s$ in $\Gamma \backslash\{q\}$. If $\gamma$ does not go through $p$, then $r$ and $s$ are connected in $\Gamma \backslash\{p, q\}$. Assume that this path $\gamma$ goes through $p$. Then $\gamma$ goes through exactly two vertices $p_{i}, p_{j}$ (we may assume $p_{1}$ and $p_{2}$ ). Moreover, one of the facets $F_{1}, F_{2}, F_{3}$, say $F_{1}$, contains both $p_{1}$ and $p_{2}$. 
Note that $F_{1}$ corresponds to the 2 -valent subgraph in $\Gamma$. Therefore, we can take the path $\gamma_{p}$ connecting $p_{1}$ and $p_{2}$ on $F_{1}$ which is not the path $p_{1} p p_{2}$. Because $p$ and $q$ are not on the same facet, in particular $q \notin V\left(F_{1}\right)$, the path $\gamma_{p}$ does not contain $q$. Hence, the connected subgraph $\gamma \cup \gamma_{p}$ contains both $r$ and $s$ but does not contain both $p$ and $q$. Thus, we can take the path $\gamma^{\prime}$ from $r$ to $s$ in $\gamma \cup \gamma_{p} \subset \Gamma \backslash\{p, q\}$. This establishes that $\Gamma \backslash\{p, q\}$ is connected.

In summary, by Lemmas 6.1 and 6.2, we have the following fact.

Corollary 6.3. Let $\Gamma$ be a one-skeleton of a 3-dimensional manifold with faces $Q$. Then, for all $p \in V(\Gamma), \Gamma \backslash\{p\}$ is connected. Furthermore, if $\Gamma \backslash\{p, q\}$ is not connected, then $p$ and $q$ are on the same facet.

\section{Proof of main theorem}

The main theorem of this paper can be stated as follows:

Theorem 7.1. Let $M$ be a simply connected 6-dimensional torus manifold with $H^{\text {odd }}(M)=0$. Then, either $M$ is equivariantly diffeomorphic to

(1) $S^{6} \subset \mathbb{C}^{3} \oplus \mathbb{R}$ with a torus action induced from a (faithful) representation of $T^{3}$ on $\mathbb{C}^{3}$,

(2) a 6-dimensional quasitoric manifold $X$, or

(3) an $S^{4}$-bundle over $S^{2}$ which is equivariantly diffeomorphic to $M(\epsilon, a, b)$ for some $\epsilon= \pm 1, a, b \in \mathbb{Z}$;

or else there are some 6-dimensional quasitoric manifolds $X_{h}$ for some $h=1, \ldots, k$, and some $S^{4}$-bundles over $S^{2}$, say $S_{i}=M\left(\epsilon_{i}, a_{i}, b_{i}\right)$ (for some $\epsilon_{i}= \pm 1, a_{i}, b_{i} \in \mathbb{Z}$ and $i=1, \ldots, \ell)$, such that $M$ is equivariantly diffeomorphic to

$$
\left(\underset{h=1}{\#} X_{h}\right) \#\left(\stackrel{\ell}{\#} S_{i}\right)
$$

where \# represents the equivariant connected sum around fixed points, $k+\ell \geq 2$ for $k \geq 0, \ell \geq 1$, and the case $k=0$ means that there is no $X_{h}$ factor.

In this final section, we prove Theorem 7.1.

Let $M$ be a simply connected 6-dimensional torus manifold with $H^{\text {odd }}(M)=0$, $Q$ be its orbit space which is homeomorphic to $D^{3}$ and $\left(\Gamma_{M}, A_{M}\right)$ be its induced oriented torus graph (we omit the orientation).

Because $\Gamma_{M}$ is a one-skeleton of a manifold with faces which is homeomorphic to $D^{3}$, it is easy to check that $\left|V\left(\Gamma_{M}\right)\right| \neq 1$, 3. If $\left|V\left(\Gamma_{M}\right)\right|=2$, by Lemma 4.1, we have that $M$ is equivariantly diffeomorphic to $S^{6}$, i.e., statement (1). If $\left|V\left(\Gamma_{M}\right)\right|=4$, it follows from Lemma 4.2 that $M$ is equivariantly diffeomorphic to a quasitoric manifold $\mathbb{C} P^{3}$ or $M(\epsilon, a, b)$ for some $\epsilon= \pm 1, a, b \in \mathbb{Z}$, i.e., statement (2) or (3) 
occurs. So we need only prove the case when $\left|V\left(\Gamma_{M}\right)\right| \geq 5$.

We first establish the following lemma.

Lemma 7.2. Assume that $\left|V\left(\Gamma_{M}\right)\right| \geq 5$ and there is a multiple edge in $\Gamma_{M}$. Then, $\left(\Gamma_{M}, A_{M}\right)$ can be decomposed as

$$
\left(\Gamma_{M}, \mathscr{A}_{M}\right)=\left(\Gamma_{X}, \mathscr{A}_{X}\right) \#\left(\Gamma_{S_{1}}, \mathscr{A}_{S_{1}}\right) \# \cdots \#\left(\Gamma_{S_{\ell^{\prime}}}, \mathscr{A}_{S_{\ell^{\prime}}}\right)
$$

or

$$
\left(\Gamma_{M}, \mathscr{A}_{M}\right)=\left(\Gamma_{S_{1}}, \mathscr{A}_{S_{1}}\right) \# \cdots \#\left(\Gamma_{S_{\ell^{\prime}}}, \mathscr{A}_{S_{\ell^{\prime}}}\right),
$$

where $\left(\Gamma_{X}, A_{X}\right)$ is a torus graph without multiple edges and $S_{i}=M\left(\epsilon_{i}, a_{i}, b_{i}\right)$ for $i=1, \ldots, \ell^{\prime}$.

Proof. Assume two vertices $p$ and $q$ are connected by a multiple edge, i.e., two edges (see the bottom graph in Figure 7). Then, by the connection of the torus graph (see Proposition 3.1), it is easy to check that the axial functions around the

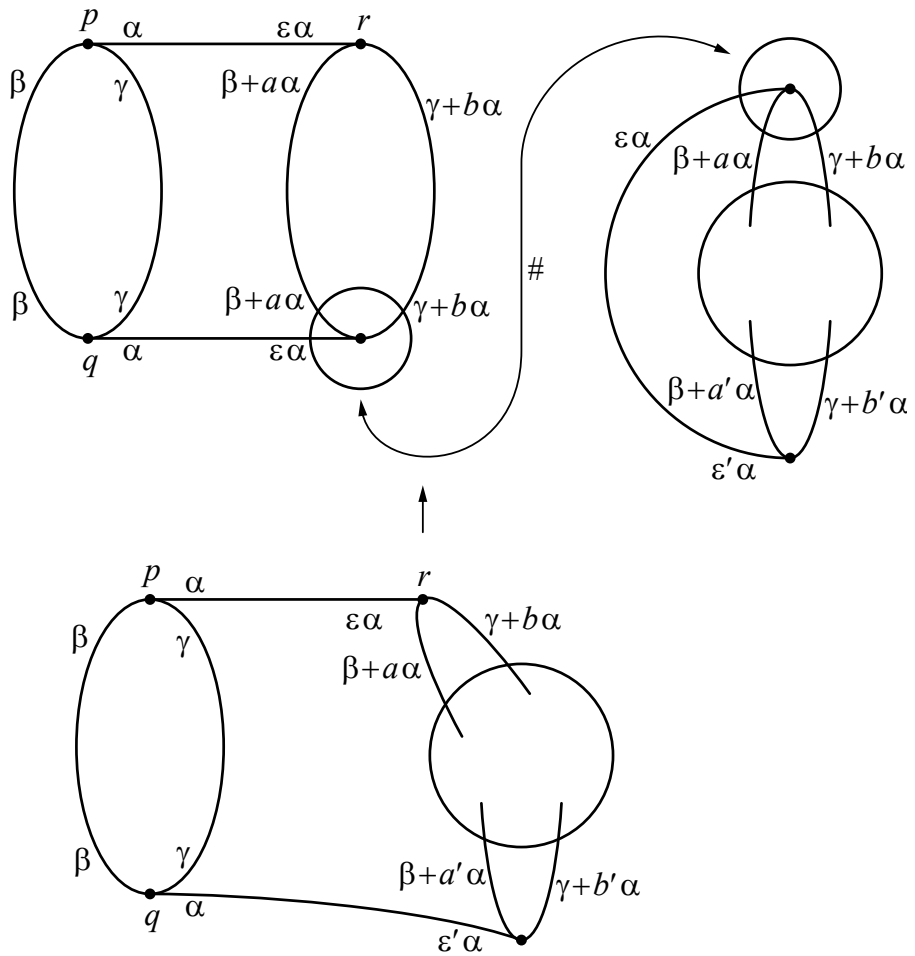

Figure 7. We may regard $\alpha, \beta, \gamma$ as any generators in $\left(\mathfrak{t}_{\mathbb{Z}}^{3}\right)^{*}$ and $a, a^{\prime}, b, b^{\prime} \in \mathbb{Z}$ and $\epsilon, \epsilon^{\prime}= \pm 1$. The bottom graph is $\left(\Gamma_{M}, \mathcal{A}_{M}\right)$, the upper-left graph is $\left(\Gamma_{S_{1}}, \mathscr{A}_{S_{1}}\right)$ and the upper-right graph is $\left(\Gamma_{M^{\prime}}, \mathscr{A}_{M^{\prime}}\right)$. If we fix the orientation of $\left(\Gamma_{M}, \mathscr{A}_{M}\right)$ then the orientations of $\left(\Gamma_{S_{1}}, \mathscr{A}_{S_{1}}\right)$ and $\left(\Gamma_{M^{\prime}}, \mathscr{A}_{M^{\prime}}\right)$ are automatically determined. 
vertex $r$ of the bottom graph in Figure 7 satisfy the axial functions expressed in that figure, where we can take $\alpha, \beta, \gamma$ as a $\mathbb{Z}$-basis of $\left(\mathfrak{t}_{\mathbb{Z}}^{3}\right)^{*}$. In this case, we can do an (inverse) connected sum such as the one expressed in Figure 7 (from the bottom to the top). Then, the induced torus graph $\left(\Gamma_{M}, A_{M}\right)$ is decomposed into two induced torus graphs $\left(\Gamma_{S_{1}}, \mathscr{A}_{S_{1}}\right)$ and $\left(\Gamma_{M^{\prime}}, \mathscr{A}_{M^{\prime}}\right)$, where $M^{\prime}$ is some simply connected 6dimensional torus manifold with $H^{\text {odd }}\left(M^{\prime}\right)=0$ by Lemma 5.1. Namely, we have

$$
\left(\Gamma_{M}, \mathscr{A}_{M}\right)=\left(\Gamma_{M^{\prime}}, \mathscr{A}_{M^{\prime}}\right) \#\left(\Gamma_{S_{1}}, \mathscr{A}_{S_{1}}\right) .
$$

If there are no multiple edges in $\Gamma_{M^{\prime}}$, then we may put $\Gamma_{M^{\prime}}=\Gamma_{X}$. Assume that there is a multiple edge in $\Gamma_{M^{\prime}}$. If there are only four vertices in $\Gamma_{M^{\prime}}$, then we may put $M^{\prime}$ as $S_{2}=M\left(\epsilon_{2}, a_{2}, b_{2}\right)$ by Lemma 4.2. When there are more than four vertices in $\Gamma_{M^{\prime}}$, we iterate the above argument, establishing the lemma.

Therefore, to prove Theorem 7.1, it is enough to prove the following lemma.

Lemma 7.3. Assume that $\left|V\left(\Gamma_{M}\right)\right| \geq 5$ and there are no multiple edges in $\Gamma_{M}$. Then, $\left(\Gamma_{M}, \mathscr{A}_{M}\right)$ can be decomposed as

$$
\left(\Gamma_{M}, \mathscr{A}_{M}\right)=\left(\Gamma_{X_{1}}, \mathscr{A}_{X_{1}}\right) \# \cdots \#\left(\Gamma_{X_{k}}, \mathscr{A}_{X_{k}}\right) \#\left(\Gamma_{S_{1}}, \mathscr{A}_{S_{1}}\right) \# \ldots \#\left(\Gamma_{S_{\ell^{\prime \prime}}}, \mathscr{A}_{S_{\ell^{\prime \prime}}}\right),
$$

where $\left(\Gamma_{X_{h}}, \mathcal{A}_{X_{h}}\right)$ for $h=1, \ldots, k$ is the torus graph induced from a quasitoric manifold $X_{h}$, and $S_{i}=M\left(\epsilon_{i}, a_{i}, b_{i}\right)$ for $i=1, \ldots, \ell^{\prime \prime}$.

Proof. If $\Gamma_{M}(=\Gamma)$ is 3-connected, then it follows from Lemma 4.4 that the statement holds, i.e., $k=1, \ell^{\prime \prime}=0$. Therefore, we may assume $\Gamma$ is not 3 -connected. In this case, by Corollary 6.3, there is a 2-valent torus subgraph $F \subset \Gamma$ such that $\Gamma \backslash\{p, q\}$ is not connected for some $p, q \in V(F)$.

If $F$ is a triangle (i.e., $|V(F)|=3$ ), using a method similar to that demonstrated in the proof of Lemma 6.1, we have that there is a face in $Q$ which has a selfintersection edge. This contradicts that $Q$ is a manifold with faces. Therefore, we may assume $|V(F)| \geq 4$. We first assume that $p q$ is an edge of $F$. Then, there are two graphs $\Gamma_{1}$ and $\Gamma_{2}$ which are the connected components of $\Gamma \backslash\{p, q\}$ expressed in Figure 8. If we remove the two vertices $r$ and $q$ from $\Gamma$ instead of $p$ and $q$, where $r \in V\left(\Gamma_{2}\right)$ such that $p r$ is an edge, then $\Gamma \backslash\{r, q\}$ is also not connected (see Figure 8 ). Therefore, we may assume that

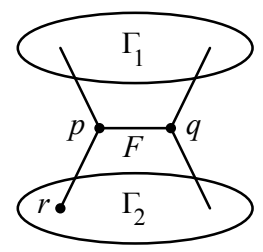

Figure 8. If we remove $r$ and $q$ from $\Gamma$ instead of $p$ and $q$, the graph is also disconnected. 
(1) $p, q \in V(\Gamma)$ are such that $\Gamma \backslash\{p, q\}$ is not connected,

(2) $p q \notin E(\Gamma)$,

(3) there is a 2-valent torus subgraph (facet) $F$ with $|V(F)| \geq 4$ in $\Gamma$ such that $p, q \in V(F)$.

We call such a facet $F$ a singular facet.

Let $F$ be a singular facet. Assume $|V(F)| \geq 6$. In this case, by an argument similar to the one just before, we may take $p$ and $q$ to be in the position of Figure 9, i.e., $p$ and $q$ are on two separated edges $r p$ and $s q$ which are common edges of two facets $F$ and $F^{\prime}$ in Figure 9 (note that $r$ and $s$ might be connected by an edge). Moreover, by considering the omnioriented characteristic functions of the facets $F$ and $F^{\prime}$, we may take the axial functions around the facet $F$ to be as in Figure 9 .

By taking an appropriate orientation, we can do the connected sum as in Figure 10; here we denote the (oriented) torus graph containing $\Gamma_{1}$ by $\left(\widetilde{\Gamma}_{1}, \widetilde{A}_{1}\right)$ and that containing $\Gamma_{2}$ by $\left(\widetilde{\Gamma}_{2}^{\prime}, \widetilde{A}_{2}^{\prime}\right)$. The torus graph obtained by this connected sum is nothing but the torus graph $(\Gamma, \mathscr{A})$ in Figure 9 . Note that $\widetilde{\Gamma}_{1}$ is simple and planar, while $\widetilde{\Gamma}_{2}^{\prime}$ is just planar. With a method similar to that demonstrated in Figure 7, $\left(\widetilde{\Gamma}_{2}^{\prime}, \widetilde{A}_{2}^{\prime}\right)$ can be obtained from the connected sum of $\left(\Gamma_{S}, \mathscr{A}_{S}\right)$ and the simple, planar graph $\left(\widetilde{\Gamma}_{2}, \widetilde{A}_{2}\right)$ (containing $\left.\Gamma_{2}\right)$, where $\left(\Gamma_{S}, \mathscr{A}_{S}\right)$ is one of the torus graphs (by taking the appropriate axial functions) in Figure 2. Namely, the torus graph in Figure 9 can be obtained from the connected sum

$$
(\Gamma, \mathscr{A})=\left(\widetilde{\Gamma}_{1}, \widetilde{\mathscr{A}}_{1}\right) \#\left(\Gamma_{S}, \mathscr{A}_{S}\right) \#\left(\widetilde{\Gamma}_{2}, \tilde{A}_{2}\right) .
$$

Here, it is easy to check that $\widetilde{\Gamma}_{i}$ consists of $\Gamma_{i}$ and the other two facets, say $\widetilde{F}(i)$ and $\widetilde{F}^{\prime}(i)$ (induced from $F$ and $F^{\prime}$ in $\Gamma$ ). Because of Figure 10, the number of

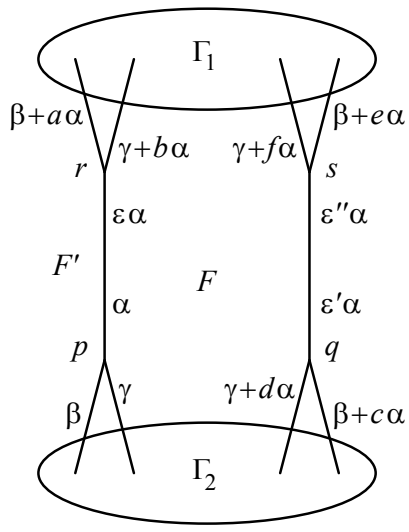

Figure 9. The axial functions around $F$ when $|V(F)| \geq 6$, where $\epsilon, \epsilon^{\prime}, \epsilon^{\prime \prime}= \pm 1$ and $a, b, c, d, e, f \in \mathbb{Z}$. Here, $F^{\prime}$ is a facet which intersects $F$ on $p r$ and $q s$. 


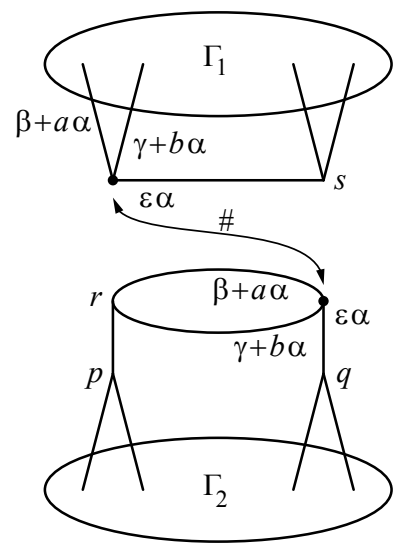

Figure 10. The torus graph $(\Gamma, \mathscr{A})$ in Figure 9 splits into two torus graphs $\left(\widetilde{\Gamma}_{1}, \widetilde{A}_{1}\right)$ (upper) and $\left(\widetilde{\Gamma}_{2}^{\prime}, \widetilde{A}_{2}^{\prime}\right)$ (lower). Here, we omit the axial functions around the vertices $p, q, r, s$ because they are exactly the same as those in Figure 9.

vertices of $\widetilde{F}(i)$ and $\widetilde{F}^{\prime}(i)$ is reduced; in particular, the number of vertices of the facet $\widetilde{F}(i)$ induced from the singular facet $F$ is strictly less than 6 . If both $\left(\widetilde{\Gamma}_{1}, \widetilde{\mathscr{A}}_{1}\right)$ and $\left(\widetilde{\Gamma}_{2}, \widetilde{A}_{2}\right)$ are 3-connected, then these torus graphs are induced from quasitoric manifolds, i.e, the statements of Lemma 7.3 hold. Assume that $\left(\widetilde{\Gamma}_{1}, \widetilde{A}_{1}\right)$ is not 3 -connected. Then, by the above arguments, there is a singular facet $F$ in $\left(\widetilde{\Gamma}_{1}, \widetilde{\mathscr{A}}_{1}\right)$. If $|V(F)| \geq 6$, then $\left(\widetilde{\Gamma}_{1}, \widetilde{A}_{1}\right)$ also decomposes as

$$
\left(\widetilde{\Gamma}_{1}, \widetilde{A}_{1}\right)=\left(\widetilde{\Gamma}_{3}, \widetilde{A}_{3}\right) \#\left(\Gamma_{S^{\prime}}, \mathscr{A}_{S^{\prime}}\right) \#\left(\widetilde{\Gamma}_{4}, \widetilde{A}_{4}\right),
$$

using arguments similar to those Figure 10. Iterating, we may reduce all singular facets with $|V(F)| \geq 6$. More precisely, we may decompose $(\Gamma, \mathscr{A})$ in Figure 9 as

$$
(\Gamma, \mathscr{A})=\#_{i=1}^{\ell}\left\{\left(\Gamma_{i}, \mathscr{A}_{i}\right) \#\left(\Gamma_{S_{i}}, \mathscr{A}_{S_{i}}\right) \#\left(\Gamma_{i+\ell}, \mathscr{A}_{i+\ell}\right)\right\}
$$

where $\left(\Gamma_{S_{i}}, \mathscr{A}_{S_{i}}\right)$ for $i=1, \ldots, \ell$ is a torus graph in Figure 2 and $\left(\Gamma_{h}, \mathscr{A}_{h}\right)$ for $h=1, \ldots, 2 \ell$ is a 3 -valent simple and planar torus graph such that either

- $\left(\Gamma_{h}, \mathscr{A}_{h}\right)$ is 3-connected (in this case, induced from a quasitoric manifold), or

- all singular facets $F$ satisfy $|V(F)|=4$ or 5 .

Assume that the number of vertices in every singular facet of the torus graph $(\Gamma, \mathscr{A})$ is less than or equal to 5 . Then, such a torus graph is one of the torus graphs expressed in Figure 11. However, because $\Gamma$ is the one-skeleton of a manifold with faces and is not 3-connected, it is easy to check that there exists a singular facet $F^{\prime}$ such that $F^{\prime} \cap F=\{p r, q s\}$ and $\left|V\left(F^{\prime}\right)\right| \geq 6$. This gives a contradiction. Hence, this case does not occur. This establishes Lemma 7.3. 

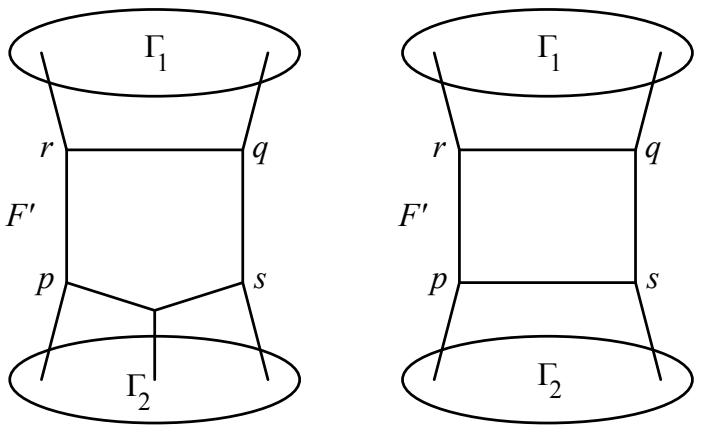

Figure 11. The singular facets $F$ with $|V(F)|=5$ or 4 . Here, $F^{\prime}$ is a facet which intersects $F$ on $p r$ and $q s$.

Consequently, by Lemmas 5.4, 7.2 and 7.3, we have the statement of Theorem 7.1. Finally, by Theorem 7.1 and the Mayer-Vietoris exact sequence, we also have the following well-known result.

Corollary 7.4. Let $M$ be a simply connected 6-dimensional torus manifold whose cohomology ring is generated by the second-degree cohomology. Then, $M$ is a quasitoric manifold.

\section{Acknowledgments}

The author would like to thank Professors Yael Karshon and Takashi Tsuboi for providing him excellent circumstances in which to do research.

\section{References}

[Buchstaber and Panov 2002] V. M. Buchstaber and T. E. Panov, Torus actions and their applications in topology and combinatorics, University Lecture Series 24, Amer. Math. Soc., Providence, RI, 2002. MR 2003e:57039 Zbl 1012.52021

[Darby 2015] A. Darby, "Torus manifolds in equivariant complex bordism", Topology Appl. 189 (2015), 31-64. MR 3342571 Zbl 06435721

[Davis and Januszkiewicz 1991] M. W. Davis and T. Januszkiewicz, "Convex polytopes, Coxeter orbifolds and torus actions”, Duke Math. J. 62:2 (1991), 417-451. MR 92i:52012 Zbl 0733.52006

[Fulton 1993] W. Fulton, Introduction to toric varieties, Annals of Mathematics Studies 131, Princeton Univ. Press, 1993. MR 94g:14028 Zbl 0813.14039

[Guillemin and Zara 2001] V. Guillemin and C. Zara, "1-skeleta, Betti numbers, and equivariant cohomology”, Duke Math. J. 107:2 (2001), 283-349. MR 2002j:53110 Zbl 1020.57013

[Hattori and Masuda 2003] A. Hattori and M. Masuda, "Theory of multi-fans", Osaka J. Math. 40:1 (2003), 1-68. MR 2004d:53103 Zbl 1034.57031

[Ishida et al. 2013] H. Ishida, Y. Fukukawa, and M. Masuda, "Topological toric manifolds", Mosc. Math. J. 13:1 (2013), 57-98. MR 3112216 Zbl 1302.53091 
[Izmest' ev 2001] I. V. Izmest' ev, "Three-dimensional manifolds defined by a coloring of the faces of a simple polytope", Mat. Zametki 69:3 (2001), 375-382. In Russian; translated in Math. Notes 69:3 (2001), 340-346. MR 2002g:57005 Zbl 0991.57016

[Kawakubo 1991] K. Kawakubo, The theory of transformation groups, Oxford Univ. Press, 1991. MR 93g:57044 Zbl 0744.57001

[Kuroki 2008] S. Kuroki, "Remarks on McGavran's paper and Nishimura's results", Trends Math. (N.S.) 10:1 (2008), 77-79.

[Kuroki 2010] S. Kuroki, "Operations on 3-dimensional small covers", Chin. Ann. Math. Ser. B 31:3 (2010), 393-410. MR 2011f:57029 Zbl 1217.57010

[Kuroki 2011] S. Kuroki, "Classification of torus manifolds with codimension one extended actions", Transform. Groups 16:2 (2011), 481-536. MR 2012g:57059 Zbl 1246.57082

[Lee 2013] J. M. Lee, Introduction to smooth manifolds, 2nd ed., Graduate Texts in Mathematics 218, Springer, New York, 2013. MR 2954043 Zbl 1258.53002

[Lü and Yu 2011] Z. Lü and L. Yu, "Topological types of 3-dimensional small covers", Forum Math. 23:2 (2011), 245-284. MR 2012h:57038 Zbl 1222.52015

[Maeda et al. 2007] H. Maeda, M. Masuda, and T. Panov, "Torus graphs and simplicial posets", Adv. Math. 212:2 (2007), 458-483. MR 2008e:55007 Zbl 1119.55004

[Masuda 1999] M. Masuda, "Unitary toric manifolds, multi-fans and equivariant index", Tohoku Math. J. (2) 51:2 (1999), 237-265. MR 2000e:57058 Zbl 0940.57037

[Masuda 2005] M. Masuda, " $h$-vectors of Gorenstein* simplicial posets", Adv. Math. 194:2 (2005), 332-344. MR 2006b:52009 Zbl 1063.05010

[Masuda and Panov 2006] M. Masuda and T. Panov, "On the cohomology of torus manifolds", Osaka J. Math. 43:3 (2006), 711-746. MR 2007j:57039 Zbl 1111.57019

[McGavran 1976] D. McGavran, " $T^{3}$-actions on simply connected 6-manifolds, I", Trans. Amer. Math. Soc. 220 (1976), 59-85. MR 54 \#3729 Zbl 0329.57019

[Nishimura 2012] Y. Nishimura, "Combinatorial constructions of three-dimensional small covers", Pacific J. Math. 256:1 (2012), 177-199. MR 2928547 Zbl 1253.57010

[Oda 1988] T. Oda, Convex bodies and algebraic geometry: an introduction to the theory of toric varieties, Ergebnisse der Mathematik und ihrer Grenzgebiete (3) 15, Springer, Berlin, 1988. MR 88m:14038 Zbl 0628.52002

[Orlik and Raymond 1970] P. Orlik and F. Raymond, "Actions of the torus on 4-manifolds, I", Trans. Amer. Math. Soc. 152 (1970), 531-559. MR 42 \#3808 Zbl 0216.20202

[Wiemeler 2013] M. Wiemeler, "Exotic torus manifolds and equivariant smooth structures on quasitoric manifolds", Math. Z. 273:3-4 (2013), 1063-1084. MR 3030690 Zbl 1269.57014

[Ziegler 1995] G. M. Ziegler, Lectures on polytopes, Graduate Texts in Mathematics 152, Springer, New York, 1995. MR 96a:52011 Zbl 0823.52002

Received September 4, 2014.

SHINTARÔ KUROKI

Graduate School of Mathematical Sciences

UNIVERSITY OF TOKYO

3-8-1 Komaba Meguro-KU

TOKYO 153-8914

JAPAN

kuroki@ms.u-tokyo.ac.jp 


\title{
PACIFIC JOURNAL OF MATHEMATICS
}

\author{
msp.org/pjm
}

Founded in 1951 by E. F. Beckenbach (1906-1982) and F. Wolf (1904-1989)

\section{EDITORS}

Don Blasius (Managing Editor)

Department of Mathematics

University of California

Los Angeles, CA 90095-1555

blasius@math.ucla.edu

\author{
Paul Balmer \\ Department of Mathematics \\ University of California \\ Los Angeles, CA 90095-1555 \\ balmer@math.ucla.edu \\ Robert Finn \\ Department of Mathematics \\ Stanford University \\ Stanford, CA 94305-2125 \\ finn@math.stanford.edu \\ Sorin Popa \\ Department of Mathematics \\ University of California \\ Los Angeles, CA 90095-1555 \\ popa@math.ucla.edu
}

\author{
Vyjayanthi Chari \\ Department of Mathematics \\ University of California \\ Riverside, CA 92521-0135 \\ chari@math.ucr.edu \\ Kefeng Liu \\ Department of Mathematics \\ University of California \\ Los Angeles, CA 90095-1555 \\ liu@math.ucla.edu \\ Jie Qing \\ Department of Mathematics \\ University of California \\ Santa Cruz, CA 95064 \\ qing@ cats.ucsc.edu
}

\section{PRODUCTION}

Silvio Levy, Scientific Editor, production@msp.org

\section{SUPPORTING INSTITUTIONS}

ACADEMIA SINICA, TAIPEI

CALIFORNIA INST. OF TECHNOLOGY

INST. DE MATEMÁTICA PURA E APLICADA

KEIO UNIVERSITY

MATH. SCIENCES RESEARCH INSTITUTE

NEW MEXICO STATE UNIV.

OREGON STATE UNIV.

\author{
STANFORD UNIVERSITY \\ UNIV. OF BRITISH COLUMBIA \\ UNIV. OF CALIFORNIA, BERKELEY \\ UNIV. OF CALIFORNIA, DAVIS \\ UNIV. OF CALIFORNIA, LOS ANGELES \\ UNIV. OF CALIFORNIA, RIVERSIDE \\ UNIV. OF CALIFORNIA, SAN DIEGO \\ UNIV. OF CALIF., SANTA BARBARA
}

\author{
Daryl Cooper \\ Department of Mathematics \\ University of California \\ Santa Barbara, CA 93106-3080 \\ cooper@math.ucsb.edu \\ Jiang-Hua Lu \\ Department of Mathematics \\ The University of Hong Kong \\ Pokfulam Rd., Hong Kong \\ jhlu@maths.hku.hk \\ Paul Yang \\ Department of Mathematics \\ Princeton University \\ Princeton NJ 08544-1000 \\ yang@math.princeton.edu
}

These supporting institutions contribute to the cost of publication of this Journal, but they are not owners or publishers and have no responsibility for its contents or policies.

See inside back cover or msp.org/pjm for submission instructions.

The subscription price for 2016 is US $\$ 440 /$ year for the electronic version, and $\$ 600 /$ year for print and electronic.

Subscriptions, requests for back issues and changes of subscribers address should be sent to Pacific Journal of Mathematics, P.O. Box 4163, Berkeley, CA 94704-0163, U.S.A. The Pacific Journal of Mathematics is indexed by Mathematical Reviews, Zentralblatt MATH, PASCAL CNRS Index, Referativnyi Zhurnal, Current Mathematical Publications and Web of Knowledge (Science Citation Index).

The Pacific Journal of Mathematics (ISSN 0030-8730) at the University of California, c/o Department of Mathematics, 798 Evans Hall \#3840, Berkeley, CA 94720-3840, is published twelve times a year. Periodical rate postage paid at Berkeley, CA 94704, and additional mailing offices. POSTMASTER: send address changes to Pacific Journal of Mathematics, P.O. Box 4163, Berkeley, CA 94704-0163.

PJM peer review and production are managed by EditFLOW ${ }^{\circledR}$ from Mathematical Sciences Publishers.

\section{PUBLISHED BY}

\section{mathematical sciences publishers \\ nonprofit scientific publishing}

http://msp.org/

(C) 2016 Mathematical Sciences Publishers 


\section{PACIFIC JOURNAL OF MATHEMATICS}

Volume $280 \quad$ No. $1 \quad$ January 2016

Stable capillary hypersurfaces in a wedge

JAIGYOUNG CHOE and MIYUKI KoISO

The Chern-Simons invariants for the double of a compression body

DAVID L. DUNCAN

Compactness and the Palais-Smale property for critical Kirchhoff equations in

closed manifolds

EMMANUEL HeBEy

On the equivalence of the definitions of volume of representations

SUNGWOON KIM

Strongly positive representations of even GSpin groups

YEANSU KIM

An Orlik-Raymond type classification of simply connected 6-dimensional torus manifolds with vanishing odd-degree cohomology

\section{SHINTARÔ KUROKI}

Solutions with large number of peaks for the supercritical Hénon equation

Zhongyuan LiU and ShuangJie Peng

Effective divisors on the projective line having small diagonals and small heights and 141 their application to adelic dynamics

YÛSUKE OKUYAMA

Computing higher Frobenius-Schur indicators in fusion categories constructed from inclusions of finite groups

\section{Peter Schauenburg}

Chordal generators and the hydrodynamic normalization for the unit ball

SEBASTIAN SCHLEISSINGER

On a question of A. Balog

ILYA D. SHKREDOV

Uniqueness result on nonnegative solutions of a large class of differential inequalities 241 on Riemannian manifolds

YUHUA SUN

Correction to "Closed orbits of a charge in a weakly exact magnetic field"

WILL J. MERRY 